\title{
Host glycosidase and flagellin glycan polymorphism control hydrolytic release of immunogenic peptides
}

Pierre Buscaill, Balakumaran Chandrasekar, Nattapong Sanguankiattichai, Jiorgos Kourelis, Farnusch Kaschani, Emma L. Thomas, Kyoko Morimoto, Markus Kaiser, Gail M. Preston, Yuki Ichinose and Renier A. L. van der Hoorn

INTRODUCTION: Immunogenic flagellin fragments are a signature of bacterial invasion in both plants and animals. Plants recognize flagellin fragments via FLS2, a model receptor kinase that is highly conserved amongst angiosperms. However, little is known about events upstream of flagellin perception by FLS2. The flagellin fragments recognized by FLS2 are buried in the flagellin polymer structure and require hydrolytic release before recognition can occur, yet the hydrolases releasing these elicitors remain to be identified. Uncovering their identity is a daunting task because the extracellular space of plants (the apoplast) is known to contain hundreds of uncharacterized glycosidases and proteases.

RATIONALE: We reasoned that pathogenic bacteria would suppress plant hydrolases that are important for immunity. We therefore monitored the activity of apoplastic hydrolases using activitybased protein profiling (ABPP) using chemical probes that irreversibly label the active site of hydrolases in an activity-dependent manner. We applied this strategy to study the infection of the tobacco relative Nicotiana benthamiana with the model bacterial pathogens Pseudomonas syringae pathovars tomato DC3000 (PtoDC3000); tabaci (Pta6605); and syringae (PsyB728a).

RESULTS: Glycosidase activity profiling of apoplastic fluids isolated from PtoDC3000-infected plants revealed that the activity of the $\beta$-galactosidase BGAL1 is suppressed in the apoplast during infection. BGAL1 suppression is caused by a heat-stable, basic, small inhibitor molecule that is produced by the bacteria under the control of the $h r p R / S / L$ virulence regulators. Null mutants of $N$. benthamiana lacking BGAL1 generated by genome editing are more susceptible, demonstrating that BGAL1 contributes to immunity. When studying why BGAL1 is suppressed, we discovered that treatment of PtoDC3000 and Pta6605 with apoplastic fluids containing BGAL1 releases an elicitor that triggers the release of reactive oxygen species (ROS) in leaf discs, a signature immune response in plants. The released elicitor is flagellin-derived because the ROS burst requires both the FLS2 receptor in the plant and flagellinencoding fliC in the bacteria. The flagellin polymer of both PtoDC3000 and Pta6605 is $O$-glycosylated with a glycan consisting of several rhamnose residues and a terminal modified viosamine (mVio). Treatment of mutant Pta6605 bacteria carrying nonglycosylated flagellin triggers the ROS burst when treated with apoplastic fluids, even in the absence of BGAL1, demonstrating that BGAL1 requires 
glycosylated flagellin to contribute to immunity. More specifically, elicitor release from rhamnosylated flagellin lacking mVio does also not require BGAL1, indicating that BGAL1 requires mVio to act in immunity. Accordingly, bgall mutant plants show enhanced susceptibility only to strains carrying mVio. Thus, BGAL1 acts in elicitor release from flagella carrying mVio glycans in Pta6605 (A) and PtoDC3000 (B). The role of BGAL1 in immunity is mitigated through the secretion of a BGAL1 inhibitor by PtoDC3000 (B), or the production of BGAL1-insensitive glycans by PsyB728a (C).

CONCLUSION: BGAL1 is an important component in the release of the flagellin elicitor in $N$. benthamiana, acting together with proteases. Bacteria use both BGAL1 inhibitors and glycan polymorphism to suppress BGAL1 function and evade recognition. Our observations indicate an important role for glycan polymorphism in bacterial pathogens of both plants and animals.

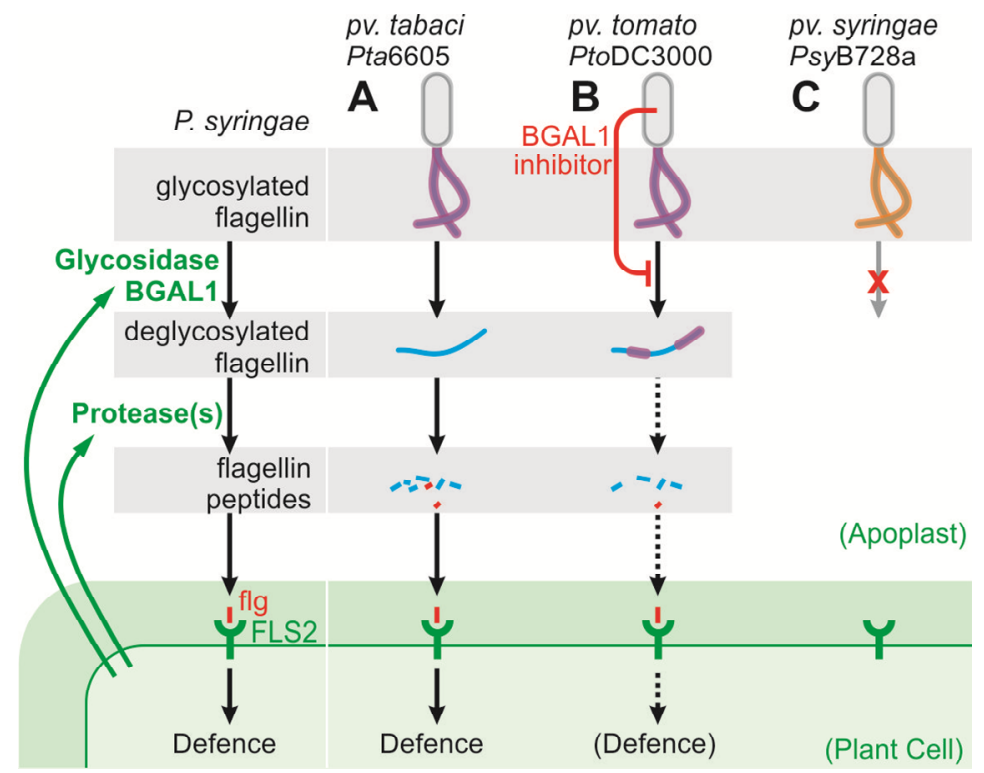

Control over hydrolytic release of immunogenic flagellin fragments. In concert with secreted proteases, $\beta$-galactosidase BGAL1 contributes to immunity against bacteria carrying glycosylated flagellin carrying terminal mVio (purple) in both Pta6605 (A) and PtoDC3000 (B) by releasing immunogenic peptides (flg, red) that are perceived by the FLS2 immune receptor. PtoDC3000 (B) mitigates BGAL1 activity by the production of a BGAL1 inhibitor, whilst PsyB728a (C) produces BGAL1-insensitive glycans to escape from recognition. 


\title{
Host glycosidase and flagellin glycan polymorphism control hydrolytic release of immunogenic peptides
}

\author{
Pierre Buscaill ${ }^{1}$, Balakumaran Chandrasekar ${ }^{1}$, Nattapong Sanguankiattichai ${ }^{1}$, Jiorgos Kourelis ${ }^{1}$, \\ Farnusch Kaschani ${ }^{2}$, Emma L. Thomas ${ }^{1}$, Kyoko Morimoto ${ }^{1}$, Markus Kaiser ${ }^{2}$, Gail M. Preston ${ }^{1}$, \\ Yuki Ichinose $^{3}$ and Renier A. L. van der Hoorn ${ }^{1 *}$ \\ ${ }^{1}$ Department of Plant Sciences, University of Oxford, Oxford, UK \\ ${ }^{2}$ ZMB Chemical Biology, Faculty of Biology, University of Duisburg-Essen, Essen, Germany \\ ${ }^{3}$ The Graduate School of Environmental and Life Science, Okayama University, Japan.
}

One sentence summary: Release of immunogenic peptides from bacterial plant pathogens is facilitated by the secreted host $\beta$-galactosidase BGAL1 and counteracted by bacterial strains carrying polymorphic glycans on flagellin or producing a BGAL1 inhibitor.

*Corresponding author: renier.vanderhoorn@plants.ox.ac.uk

\section{ABSTRACT ( $<125$ words) 125}

Plants and animals recognise conserved flagellin fragments as a signature of bacterial invasion. These immunogenic elicitor peptides are embedded in the flagellin polymer and require hydrolytic release before they can activate cell surface receptors. Although much of flagellin signalling is understood, we know little on the release of immunogenic fragments. Here we discovered that host-secreted $\beta$-galactosidase-1 (BGAL1) promotes hydrolytic elicitor release and acts in immunity against pathogenic Pseudomonas syringae strains only when they carry a terminal modified viosamine ( $\mathrm{mVio}$ ) in the flagellin $O$-glycan. In counter defence, $P$. syringae pathovars evade host immunity by using BGAL1-resistant $O$-glycans or by producing a BGAL1 inhibitor. Polymorphic glycans on flagella are common to plant and animal pathogenic bacteria and represent an important determinant of host immunity to bacterial pathogens.

Plant pathogenic bacteria colonise the extracellular space within plant tissues (the apoplast) and manipulate their hosts with toxins and effector proteins delivered through the type-III secretion system (T3SS) (1). Whereas these manipulation events inside the host cell are well studied, less is known about how bacteria manipulate the host apoplast.

One crucial aspect of plant-bacteria interactions is the recognition of extracellular pathogenassociated microbial patterns (PAMPs) that the plant can perceive through pattern recognition receptors 
(PRRs) to mount PAMP-triggered immunity (PTI) (2). Most plants are able to recognize immunogenic peptides of bacterial flagellin through homologs of FLS2, a receptor kinase PRR (2-3). The 22 amino acid flagellin fragment flg22 has been intensively used to study RLK signalling and PTI in plants. Many T3SS-secreted effectors interfere in FLS2 signalling, underlining the important role of FLS2 signalling in immunity (3-4). However, the release of immunogenic peptides like flg22 from the flagellin precursor protein is unknown, yet anticipated because these peptides are buried in the flagellin polymer structure (5). Identification of elicitor-releasing hydrolases is a daunting task because the plant apoplast contains hundreds of glycosidases, proteases and other hydrolases (6-7).

Here, we set out to discover apoplastic hydrolases involved in immunity under the assumption that harmful host-secreted hydrolases will be suppressed by these pathogens. To study apoplast manipulation, we investigated the interaction between Nicotiana benthamiana and the model bacterial pathogen Pseudomonas syringae pv. tomato DC3000 (PtoDC3000) (8). While PtoDC3000 triggers nonhost resistance via recognition of the hopQ1-1 effector, the mutant lacking this type-III effector (PtoDC3000 $(\Delta h Q))$ is virulent on $N$. benthamiana (8).

\section{Results}

\section{BGAL1 is inhibited during infection}

To monitor the suppression of extracellular glycosidases, we applied glycosidase activity profiling on apoplastic fluids isolated from infected plants and mock-inoculated control plants. Using the fluorescent activity-based probe JJB70, which labels the active site of glycosidases (9-10), we discovered a 19fold suppression of JJB70 labeling of a $45 \mathrm{kDa}$ protein, previously identified as a putative $\beta$ galactosidase (BGAL1, NbS00024332g0007 (10)) (Fig. 1A). BGAL1 carries a signal peptide and is enriched in the apoplast (fig. S1). BGAL1 also accumulates in the apoplast when transiently overexpressed by infiltration of $N$. benthamiana with Agrobacterium tumefaciens (agroinfiltration) (Fig. 1B).

BGAL1 is indeed a functional $\beta$-galactosidase because it can cleave galactose from FDG (fluorescein di- $\beta$-D-galactopyranoside, fig. S2), and both JJB70 labeling and FDG hydrolysis by BGAL1 are blocked by the $\beta$-galactosidase inhibitor galactostatin (GSTN, Fig. 1B). BGAL1 also selectively catalyses hydrolyses of 4-nitrophenyl- $\beta$-galactopyranoside but not other monosaccharide conjugates (fig. S3). In contrast to active BGAL1, mutant BGAL1 protein carrying mutated catalytic residues (BGAL1 ${ }^{\mathrm{E} 183 \mathrm{~A}, \mathrm{E} 254 \mathrm{~A}}$ ) does not accumulate upon agroinfiltration (Fig. 1B). This nonaccumulating mutant BGAL1 is used as a 'minus BGAL' (-BGAL1) control in follow up experiments. Consistent with the strong suppression of JJB70 labeling in infected plants, FDG-hydrolysing activity is $\sim 30$-fold reduced in apoplastic fluids upon infection (Fig. 1C).

Quantitative proteomic analysis indicated no change in the amounts of BGAL1 protein in apoplastic fluids of infected plants (fig. S4), suggesting that BGAL1 is inactivated during infection. To test if apoplastic fluids of infected plants contains a BGAL1 inhibitor, we mixed apoplastic fluids from 
infected and non-infected plants before and after JJB70 labeling, a method we have coined 'Convolution ABPP' (Activity-based Protein Profiling (11)). Apoplastic fluids from infected plants could suppress both JJB70 labeling (Fig. 1A) and FDG-hydrolysing activity (Fig. 1C) present in apoplastic fluids from the mock control, indicating that apoplastic fluids from infected plants contain a BGAL1 inhibitor (Fig. 1D). The BGAL1 inhibitor is detected early during infection (fig. S6A) and also when $P$ toDC3000 $(\Delta h Q)$ is grown in T3SS-inducing minimal media (fig. S5B). Inhibitor production is absent in $\Delta h r p R, \Delta h r p S$ and $\Delta h r p L$ mutants but present in $\triangle h r p A$ and $\Delta h r c C$ mutants of PtoDC3000, demonstrating that BGAL1 inhibitor production is controlled by virulence regulators but does not require the T3SS system (fig. S5B). The BGAL1 inhibitor is a heat-stable molecule of $<3 \mathrm{kDa}$ (fig. S6A), that is hydrophilic and basic (fig. S6B and S6C). This BGAL1 inhibitor does not suppress the activity profiles of other hydrolases (fig. S6D) but remains to be identified.

\section{BGAL1 contributes to immunity}

We next investigated why PtoDC3000 inhibits BGAL1. BGAL1 is one of the 28 putative Glycosyl Hydrolases of family 35 (GH35) of $N$. benthamiana (fig. S7). N. benthamiana is an ancient alloploid, and BGAL1 has one putative homeolog, which has been pseudogenized (fig. S8A). Using genome editing with CRISPR/Cas9 (12), we generated two independent BGAL1 null mutants: bgall-1 and bgal1-2 (Fig. 2A and fig. S8). Both bgall mutants contain frameshift mutations in the BGAL1 open reading frame (fig. S8B) and BGAL1 depletion causes no major growth or developmental phenotypes (Fig. 2B). Apoplastic fluids from both bgall mutants lack the JJB70 signal corresponding to BGAL1 (Fig. 2C), and have strongly reduced FDG hydrolysing activity (Fig. 2D). Upon spray inoculation with PtoDC3000( $\Delta h Q)$, both bgall mutants support higher bacterial growth (Fig. 2E), and develop more disease symptoms (Fig. 2F), confirming a role for BGAL1 in immunity. A similar increase in susceptibility was observed upon depletion of $B G A L 1$ transcripts using virus-induced gene silencing (VIGS, fig. S9).

\section{BGAL1 facilitates release of flagellin elicitor}

We hypothesized that BGAL1 contributes to immunity by releasing an elicitor from bacteria that triggers a burst of reactive oxygen species (ROS), a signature immune response in plants. We therefore established an elicitor-release assay based on the incubation of bacteria with apoplastic fluids, and detection of released elicitors in a ROS burst assay using leaf discs of unchallenged plants (Fig. 3A). The ROS burst was induced in leaf discs of $N$. benthamiana when treated with bacteria that were incubated with apoplastic fluid from leaves overexpressing BGAL1 (Fig. 3B). The ROS burst was also absent when bacteria were omitted (fig. S10), indicating that the elicitor is derived from the bacteria. A similar BGAL1-dependent ROS burst was triggered in leaf discs of Arabidopsis thaliana (Fig. 3C), indicating that the BGAL1-released elicitor is recognized by different plant families. 
We next found that Arabidopsis mutants lacking glycan receptors LORE, CERK1 and LYM1/LYM3 still perceive the elicitor (fig. S11), indicating that the BGAL1-released elicitor is not related to lipopolysaccharide, chitin or peptidoglycan, respectively (13-15). The ROS burst was also unaffected in leaf discs lacking SOBIR1 (fig S11D), but was absent in leaf discs lacking BAK1 (Fig. 3D), indicating that elicitor perception involves a membrane-localised receptor-like kinase (RLK) but not a receptor-like protein (16). When testing BAK1-dependent immune RLKs, we discovered that leaf discs of the Arabidopsis mutant lacking FLS2, the receptor-like kinase mediating flagellin recognition (17), are blind to the BGAL1-released elicitor (Fig. 3E). Likewise, N. benthamiana plants silenced for the FLS2 ortholog (NbFls2 (18)) were unable to sense the BGAL1-released elicitor (Fig. 3F). By contrast, an Arabidopsis mutant lacking EFR, the receptor for fragments of the bacterial EF-Tu protein, still perceives the BGAL1-released elicitor (fig. S11E).

Consistent with flagellin recognition via FLS2, BGAL1 treatment of mutant bacteria lacking flagellin (PtoDC3000( $\Delta h Q \Delta f l i C(19))$, did not trigger the ROS burst in Arabidopsis (Fig. 3G) or $N$. benthamiana (Fig. 3H), indicating that the BGAL1-released elicitor is derived from flagellin. Indeed, treatment of purified flagellin with apoplastic fluids containing BGAL1 also releases the elicitor (Fig. 3I), demonstrating that BGAL1 facilitates elicitor release directly from flagellin. Mass spectrometric analysis of peptides released from purified flagellin by apoplastic fluids containing BGAL1 revealed several peptides known to trigger immunity (20) (fig. S12). These data demonstrate that BGAL1 controls the release of immunogenic peptides from flagellin.

\section{BGAL1 act on glycosylated flagellin}

A conserved fragment of 22 amino acid residues of flagellin (flg22) containing the immunogenic sequence KINSAKDDAAGLQ (flg15- $\Delta 2(20)$ ), is universally recognized in angiosperms by orthologs of FLS2 (20-23). This immunogenic region resides inside the flagellin rod (Fig. 4 and fig. S13) and has to be unfolded and excised before it can interact with FLS2 $(5,24)$. The solvent-exposed surface of flagella are covered with $O$-glycans linked to six serine residues located in the D2 and D3 domains of each flagellin protein (Fig. 4). The $O$-glycans of PtoDC3000 consist of two or three rhamnose residues and a terminal modified viosamine (mVio) (25-26) (Fig. 4).

The involvement of BGAL1 in the release of the flagellin elicitor suggests that BGAL1 acts on glycosylated flagellin, and that glycan removal exposes the flagellin protein to proteases that release the flagellin elicitor (Fig. 5). We tested this hypothesis using P. syringae. pv. tabaci 6605 (Pta6605), which is also pathogenic on $N$. benthamiana (27) and has been used as a model to elucidate the glycan structure on the flagellin. The $O$-glycans on the flagellin of Pta6605 are identical to those of PtoDC3000 (Fig. 6A (25-26, 28)), but Pta6605 does not produce a BGAL1 inhibitor (fig. S14). As with PtoDC3000( $\Delta h Q)$, BGAL1-treatment of Pta6605 bacteria releases an elicitor detected in both $N$. benthamiana (Fig. 6B) and Arabidopsis (fig. S15A) and Pta6605 grows better on N. benthamiana bgall mutants than on WT plants (Fig. 6C). The elicitor released by BGAL1 is derived from flagellin as it is 
undetected in $N$. benthamiana and Arabidopsis lacking NbFLS2 and FLS2, respectively (fig. S15B-C), and the elicitor is not released from Pta6605 $(\Delta f l i C)$ lacking flagellin (fig. S15D).

Next, we took advantage of Pta6605 mutants that lack different enzymes involved in the $O$ glycan formation on flagellin. Incubation of Pta6605( $\Delta f g t l)$ bacteria, which carries unglycosylated flagellin (Fig. 6D (28)), with apoplastic fluids releases the elicitor, irrespective of BGAL1 presence (Fig. 6E). Accordingly, Pta6605( $\Delta f g t 1)$ bacteria grew less when compared to Pta6605(WT), and BGAL1 no longer contributed to immunity because $\Delta f g t 1$ bacteria grew equally well on both wild-type plants and bgall mutant plants (Fig. 6F). Pta6605 mutants producing flagella that lack one $O$ glycosylation site (S143A, S164A, S176A, S183A, S193A, and S201A mutants, respectively (28)) also released the elicitor when incubated with apoplastic fluids, even in the absence of BGAL1 (fig. S16). Consistent with the implicated role of proteases in the release of flagellin fragments, elicitor release from Pta6605( $\Delta f g t 1)$ bacteria and purified nonglycosylated flagellin was blocked with a protease inhibitor cocktail (fig. S17). These data demonstrate that $O$-glycans on flagellin provide protection against hydrolytic elicitor release and that BGAL1 overcomes this protection.

\section{BGAL1 role is specific for $m$ Vio glycans}

To further specify BGAL1 action, we tested the Pta6605 mVio transferase mutant $\Delta v i o T$ and mVio biosynthesis mutants $\triangle v i o A$ and $\triangle v i o B$, which all carry flagellin with rhamnose $O$-glycans that lack the terminal mVio residue (Fig. 6G (29)). Like the $\Delta f g t 1$ mutant, incubation of $\Delta v i o T, \Delta v i o A$, and $\Delta v i o B$ mutant bacteria with apoplastic fluids released the flagellin elicitor irrespective of BGAL1 presence (Fig. 6H and fig. S18) and these mutant bacteria grew equally well on wild-type and bgall mutant plants (Fig. 6I and fig. S18). These data demonstrate that rhamnosyl glycans on flagella do not prevent elicitor release in the apoplast and indicate that $\mathrm{mVio}$ protects against elicitor release. These data also show that BGAL1-mediated elicitor release and the role of BGAL1 in immunity requires mVio on the flagellin glycans.

All $P$. syringae bacteria produce $O$-glycosylated flagella but the structures of the $O$-glycans can differ between strains. For instance, P. syringae pv. syringae B728a (PsyB728a), which has been isolated from bean but is also pathogenic on $N$. benthamiana (30), does not carry a $(1,3)$-linked mVio but instead carries a putative (1,2)-linked terminal GlcNac (N-acetylglucosamine, Fig. 6J (25-26)). Treatment of PsyB728a with apoplastic fluids containing BGAL1 does not release the flagellin elicitor (Fig. 6K) and PsyB728a also grows equally well on wild-type and bgall mutant plants (Fig. 6L). PsyB728a does not produce a BGAL1 inhibitor (fig. S14) and may suppress elicitor release through other mechanisms. These data indicate that the flagellin glycan of PsyB728a is protected against BGAL1 from N. benthamiana. However, plants silenced for NbFls2 are more susceptible to PsyB728a (18), which indicates that $N$. benthamiana has hydrolases that can release the flagellin elicitor from PsyB728a. We did not detect this activity in our elicitor-release assays, possibly because these hydrolases are not 
sufficiently present or active in the isolated apoplastic fluids used. Overexpression of these hydrolases might overcome this limitation, as we discovered for BGAL1.

\section{Discussion}

\section{Hydrolytic release of immunogenic peptides}

We discovered that BGAL1 acts in immunity by controlling the release of immunogenic flagellin peptides, and that bacteria counter the role of BGAL1 by producing a BGAL1 inhibitor or BGAL1insensitive glycans. Our work also implicates an important role for proteases in the release of immunogenic peptides. The $N$. benthamiana apoplast contains nearly 200 proteases that may act redundantly (7) and our strategy to identify hydrolases that are suppressed during infection might be useful to identify the proteases that act in the release of flagellin elicitor. We anticipate a similar important role for BGAL1 and other hydrolases in the release of different immunogenic flagellin fragments that are recognized in tomato and rice (31-32). Hydrolytic release of immunogenic peptides from precursors may also be required for peptide elicitors elf18, nlp24 and possibly pep13 from bacterial, fungal and oomycete pathogens because these are also buried within folded protein structures (33-37). It will be interesting to determine if the role of BGAL1 and proteases is conserved across plant species or if host-specific hydrolytic processes dictate elicitor release and host specificity.

\section{Glycan polymorphism as common defence strategy}

That PsyB728a evades flagellin hydrolysis and recognition by the host suggests that polymorphic glycans on flagellin might help other plant-pathogenic bacteria to evade recognition. Indeed, the 2150 Da glycosylation of flagellin in strain $\mathrm{K} 1$ of the bacterial rice pathogen Acidovorax avenae (AcK1) prevents flagellin recognition in rice, whereas the 1600 Da glycosylation on flagellin from the related $A c \mathrm{~N} 1141$ strain does not prevent flagellin recognition (38). Likewise, despite being identical in protein sequence, the glycosylated flagellin of $P$. syringae pv. glycinea $(P s g)$ triggers cell death in tobacco $(N$. tabacum) but the glycosylated flagellin of Pta6605 does not, probably because of their distinct $O$ glycans (39-40). These observations indicate that polymorphic glycans are an important determinant in evading host immunity and that host plants secrete specialised glycosidases to release immunogenic flagellin fragments.

The role of flagellin glycans in host immunity evasion also extends to animal-pathogenic bacteria. Flagellin glycosylation mutants of $P$. aeruginosa, for instance, are less virulent in wound infection assays on mouse (41). Likewise, nonglycosylated flagellin proteins from the opportunistic human pathogen Burkholderia cenocepacia stimulate stronger TLR5-dependent immune responses in human cell lines when compared to glycosylated flagellin (42). Glycans on flagellin are also polymorphic in animal-pathogenic bacteria (43). For instance, B. cenocepacia, B. pseudomallei and B. thailandensis carry different glycans that may provide different levels of protection against host 
hydrolases (44). These data indicate that polymorphic glycans on flagella are a common strategy to evade host immunity driven by host-specific hydrolases in both plants and animals.
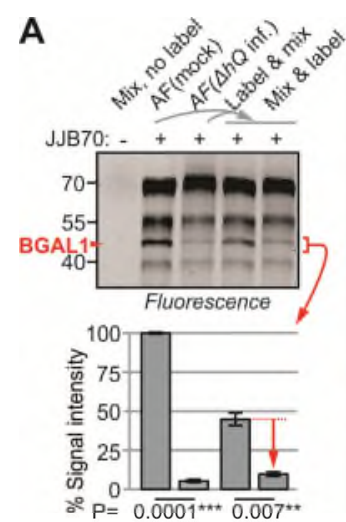

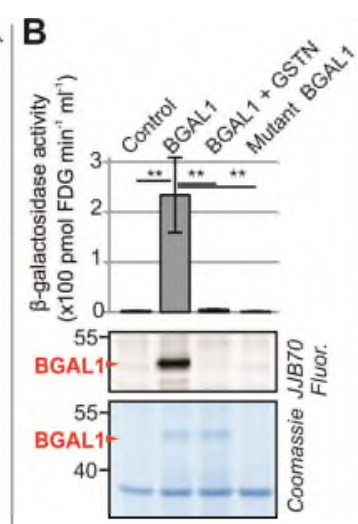

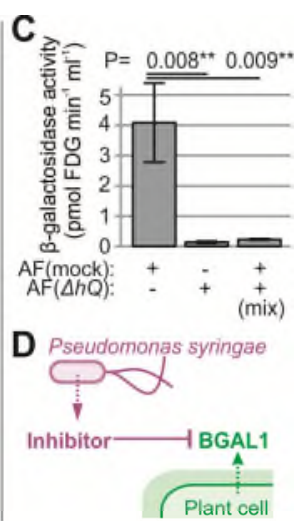

\section{Fig. 1. BGAL1 is suppressed during bacterial infection.}

(A) BGAL1 labeling is suppressed in apoplastic fluids of infected plants at 2 days post infection (dpi). The two samples were also mixed 1:1 before (mix \& label) and after (label \& mix) labeling with JJB70. The quantified fluorescence of the BGAL1 signal is plotted below. Error bars, mean \pm SE of $n=3$ replicates, $t$-test $\mathrm{P}$ values. (B) BGAL1 has $\beta$-galactosidase activity. FDG-hydrolysing activity was measured on apoplastic fluids isolated from leaves transiently expressing silencing inhibitor P19 alone (control) or with BGAL1 (+) or mutant BGAL1 (-), pre-incubated with/out $10 \mu \mathrm{M}$ galactostatin (GSTN). Error bars: mean $\pm S E$ of $n=4$ replicates; Tukey HSD P values. The samples were also labeled with JJB70 and stained with coomassie. (C) Reduced $\beta$-galactosidase activity in apoplastic fluid from infected plants isolated at $2 \mathrm{dpi}$. The two samples were also mixed 1:1 before adding FDG. Error bars: mean $\pm \mathrm{SE}$ of $n=5$ replicates; Tukey HSD P values. (D) Schematic diagram showing BGAL1 and its inhibitor. 

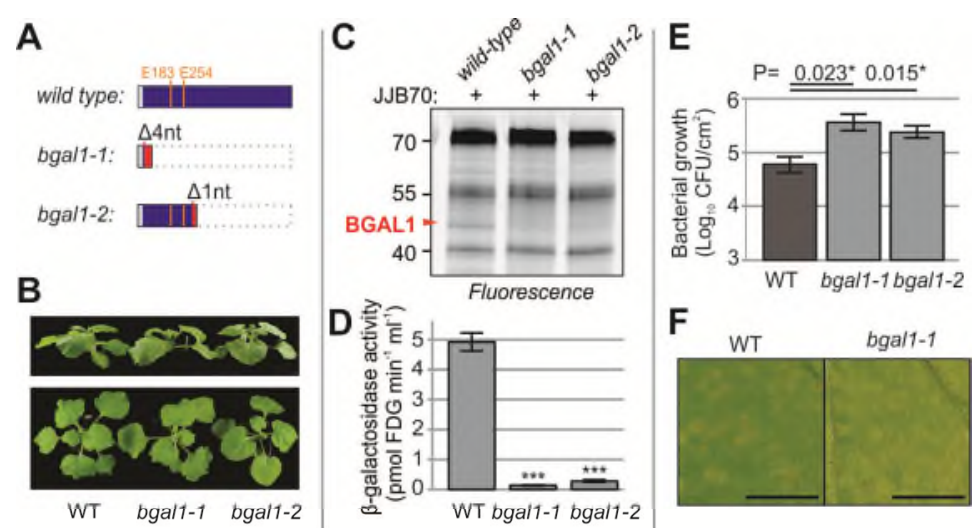

\section{Fig. 2. BGAL1 contributes to immunity}

(A) Open reading frames of wild-type and two independent bgall null mutants generated by genome editing (red, out-of-frame translation; orange, catalytic Glu residues). (B) Representative pictures of 5week old plants. (C) Both bgall null mutants lack the BGAL1 signal in JJB70 labeling profile. (D) Apoplastic fluid from both bgal mutants have reduced $\beta$-galactosidase activity. Error bars: mean $\pm \mathrm{SE}$ of $\mathrm{n}=3$ replicates; Tukey HSD P values $<0.001(* * *)$. (E) Both bgall mutants are less resistant to $\operatorname{PtoDC} 3000(\Delta h Q)$. Bacterial growth of $\operatorname{PtoDC} 3000(\Delta h Q)$ upon spray inoculation was measured at 3 dpi. Error bars: mean $\pm \mathrm{SE}$ of $\mathrm{n}=4$ replicates; $t$-test $\mathrm{P}$ values. $(\mathbf{F})$ Representative picture of bacterial spot disease symptoms taken from spray-infected leaves at 6 dpi. Size bar $=1 \mathrm{~cm}$. 
A
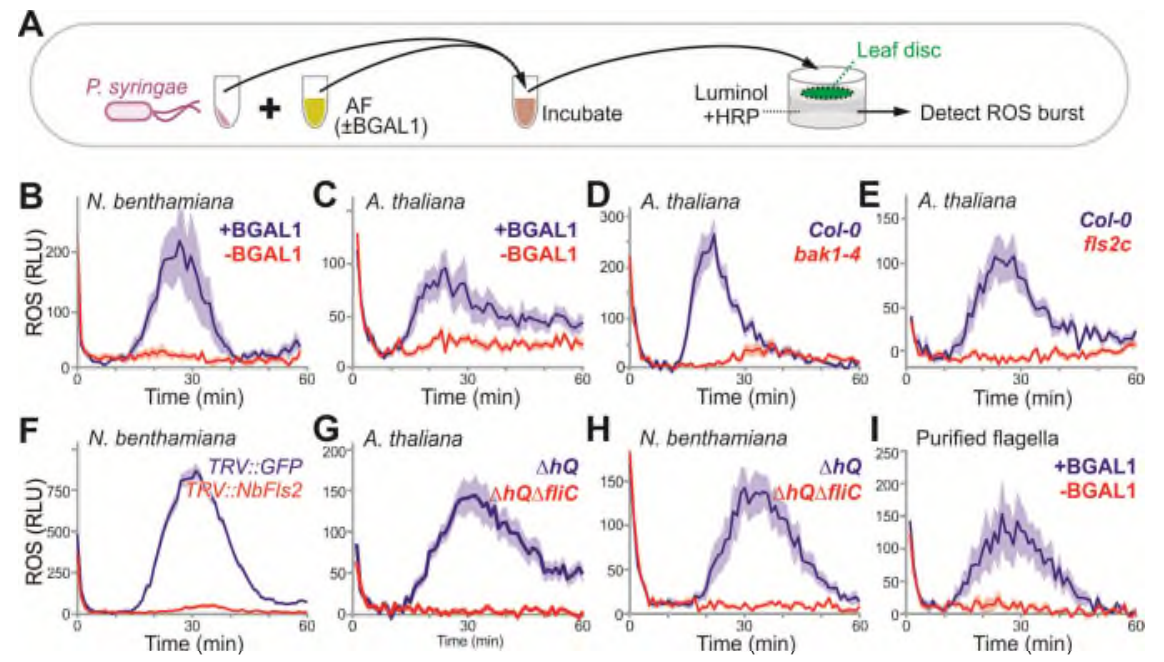

Fig. 3. BGAL1 controls the release of the flagellin elicitor.

(A) Experimental procedure to detect BGAL1-released elicitors from bacteria. The pellet of a bacterial culture was resuspended and incubated with apoplastic fluid (AF) isolated from $N$. benthamiana leaves transiently overexpressing BGAL1 (+) or the minus BGAL control (-). The treated bacteria were added to leaf discs floating on a solution containing luminol and horse-radish peroxidase (HRP) and ROS was detected over time by chemiluminescence. The ROS burst is triggered in leaf discs of N. benthamiana (B) and Arabidopsis (C) following pre-treatment of bacteria with apoplastic fluids from plants overexpressing BGAL1 (+BGAL1) but not the nonaccumulating mutant BGAL1 (-BGAL1). This ROS burst requires BAK1 (D) and FLS2 (E) and its ortholog NbFLS2 in $N$. benthamiana (F). The ROS burst also requires the flagellin-encoding $f l i C$ gene in PtoDC3000 in both Arabidopsis (G) and $N$. benthamiana $(\mathbf{H})$, and is also induced in $N$. benthamiana by purified flagella treated with AF containing BGAL1 (I). Error intervals: mean $\pm \mathrm{SE}$ of $\mathrm{n}=6$ (B, I) or $\mathrm{n}=12(\mathrm{C}-\mathrm{H})$ replicates. RLU, relative luminescence units. 

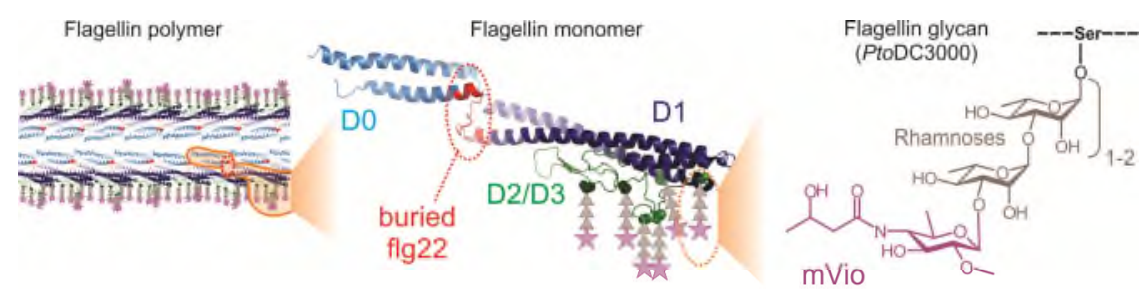

Fig. 4. Flagellin is a glycosylated protein polymer with a buried immunogenic sequence. Flagellin of PtoDC3000 was modelled based on the flagellin polymer structure of P. aeruginosa (5wk5). Positions of $O$-glycosylated Ser residues (green) are distributed over the surface of the polymer. 


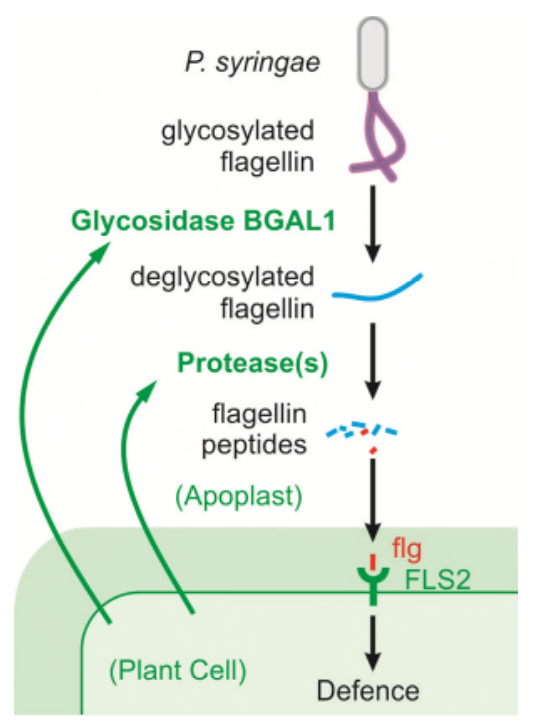

Fig. 5. Hydrolytic release of immunogenic peptides from flagellin. We hypothesize that BGAL1 controls the release of immunogenic flagellin elicitors (e.g. flg, red) from P. syringae by initiating the removal of the glycan protection layer from flagella. Next, proteases would process flagellin to release of the peptide elicitor, which are recognized by the FLS2 surface receptor. 

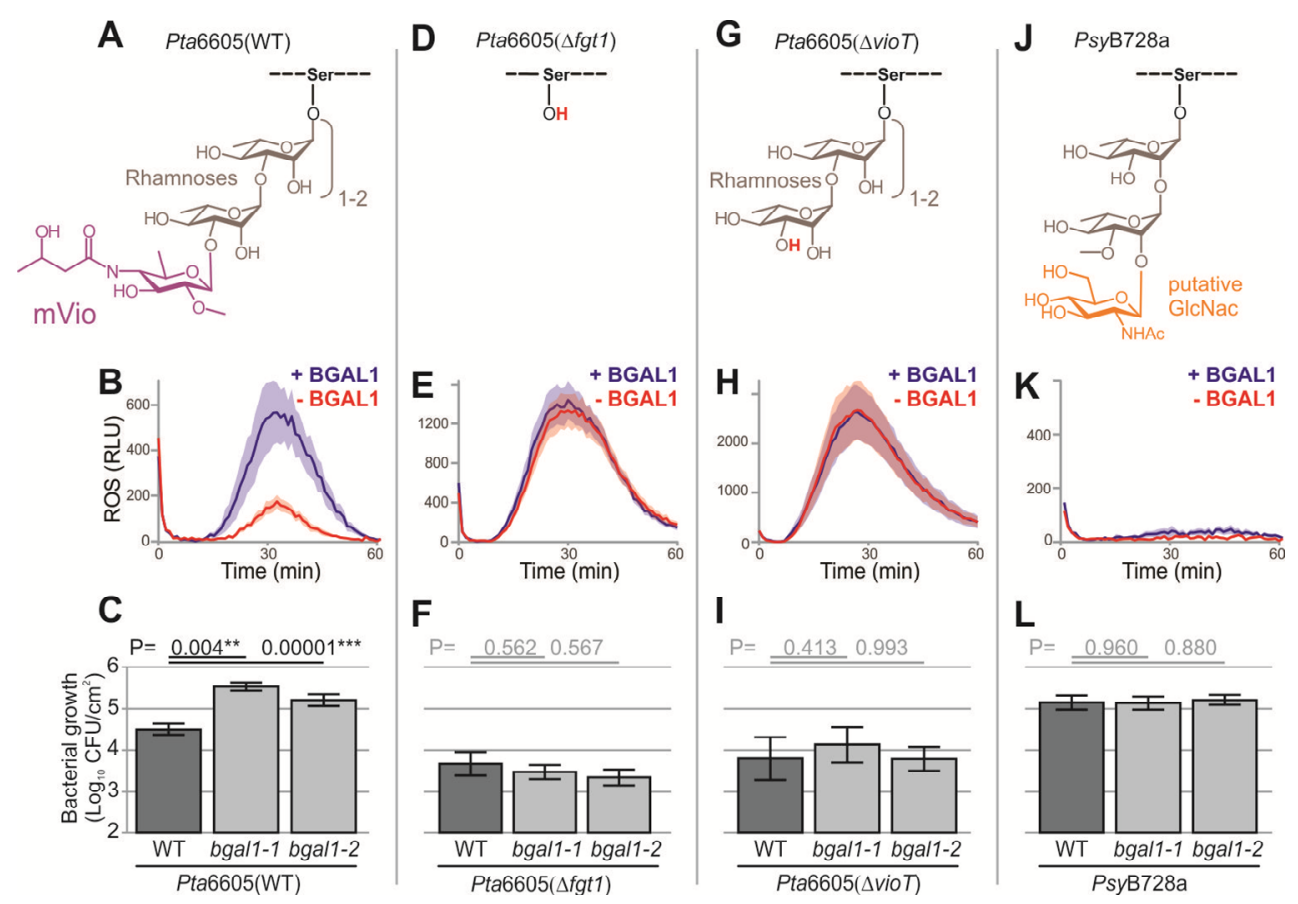

Fig. 6. BGAL1 acts in immunity only on bacteria carrying mVio on flagellin O-glycans.

(A-C) BGAL1 also releases an elicitor from Pta6605. (D-F) BGAL1 is not required to release the flagellin elicitor from Pta6605( $\Delta f g t 1)$ producing nonglycosylated flagellin. (G-I), BGAL1 is not required to release the flagellin elicitor from Pta6605( $\Delta$ vioT) lacking the terminal mVio. (J-L) BGAL1 does not release flagellin elicitor from PsyB728a carrying a different flagellin glycan. (A, D, G, J), structures of flagellin $O$-glycans, determined previously. (B, E, H, K), ROS burst assays of elicitor fractions from bacteria treated with apoplastic fluids from plants over expressing BGAL1 (+BGAL1) or mutant BGAL1 (-BGAL1). Error intervals: mean \pm SE of $n=12$ replicates. (C, F, I, L), Pta6605 growth at $3 \mathrm{dpi}$ on WT and bgall mutant plants following spray inoculation. Error bars: mean $\pm \mathrm{SE}$ of $\mathrm{n}=3$ (C), $\mathrm{n}=6(\mathrm{D})$ or $\mathrm{n}=8(\mathrm{~B}, \mathrm{E})$ replicates; $t$-test P-values. 


\section{ACKNOWLEDGEMENTS}

We thank Matthieu Joosten and Andrea Gust for providing seeds of Arabidopsis mutants; Hermen Overkleeft for providing glycosidase probes; Imre Somssich for providing flg22; Cyril Zipfel for excellent suggestions and providing fls $2 c$ seeds; Alan Collmer for providing PtoDC3000 mutants; Ursula Pyzio for plant care; John Baker for photography; Jonathan Jones, Sophien Kamoun and Sylvestre Marillonnet for providing plasmids via AddGene; and Réka Tóth and Daniela Sueldo for critically reading the manuscript. Funding: ERC Consolidator grant 616447 'GreenProteases' (PB, KM, RH); BBSRC grants BB/R017913/1 (PB, RH); the Royal Thai Government Scholarship (NS); the Clarendon foundation (JK); the Oxford Interdisciplinary Bioscience DTP (BB/M011224/1 to NS, ET, GP). Author contributions: PB, BC, NS, GP and RH conceived the project; PB, BC, NS, ET and KM performed experiments; JK performed bioinformatic analysis; FK and MK performed proteomic analysis; YI provided materials; RH wrote the manuscript with input from all authors. Competing interests: The authors declare no competing financial interests. Data and materials availability: all data is available in the manuscript, the supplementary materials, and the cited references. The proteomics data have been deposited to the ProteomeXchange Consortium via the PRIDE partner repository (https://www.ebi.ac.uk/pride/archive/) with dataset identifiers PXD010461 and PXD011823.

\section{List of Supplementary Materials}

Figure S1 BGAL1 is a secreted protein.

Figure S2 Assay for $\beta$-galactosidase activity.

Figure S3 Further characterization of BGAL1 activity.

Figure S4 BGAL1 protein level is unaltered upon infection.

Figure S5 Characterization of BGAL1 inhibitor production by the bacteria.

Figure $\mathbf{S 6}$ Further characterization of the BGAL1 inhibitor.

Figure S7 Molecular phylogenetic analysis of BGAL1 homologs.

Figure $\mathbf{S 8}$ The two independent BGAL1 mutants grow and develop normally.

Figure S9 BGAL1 depletion by VIGS enhances susceptibility to $P$. syringae.

Figure S10 No ROS burst is triggered by apoplastic fluids in the absence of bacteria.

Figure S11 Arabidopsis sd1-29, cerk1, lym1/lym3, sobir1 and efr-1 mutants still perceive the BGAL1-released elicitor.

Figure S12 Immunogenic peptides are released by apoplastic fluids containing BGAL1.

Figure $\mathbf{S 1 3} \mathrm{flg} 22$ peptide is folded and buried inside the glycosylated flagellin polymer.

Figure S14 Pta6605 and PsyB728a do not produce a BGAL1 inhibitor.

Figure S15 BGAL1 also releases the flagellin elicitor from Pta6605.

Figure $\mathbf{S 1 6}$ Single site glycan mutants trigger a BGAL1-independent ROS burst.

Figure S17 Protease inhibitor cocktail prevents elicitor release. 
Figure S18 Pta6605 $\triangle v i o A$ and $\triangle v i o B$ mutants release elicitor irrespective of BGAL1 treatment. 
Supplemental figures Buscaill et al., Host glycosidase and flagellin glycan polymorphism control hydrolytic release of immunogenic peptides

A
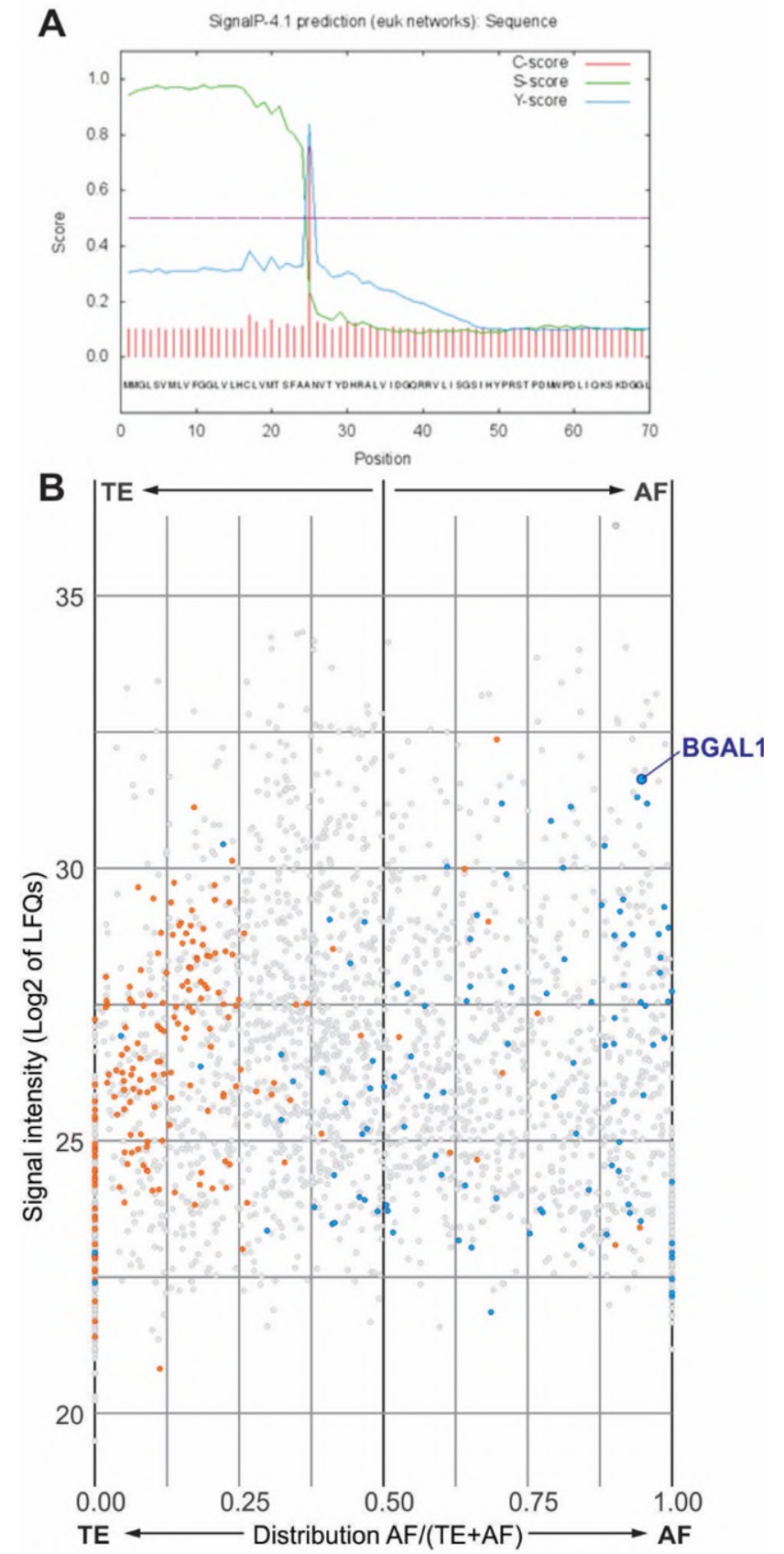

- GO:0003735 (molecular function: structural constituent of ribosome) - GO:0016798 (molecular function: hydrolase activity, acting on glycosyl bonds) All other GO terms
Figure $\mathrm{S} 1 \mathrm{BGAL} 1$ is a secreted protein. (A) SignalP score for the BGAL1 protein sequence. The BGAL1 protein sequence was analyzed by SignalP version 4.1 (45). Overall SignalP Score $=0.889$. $($ B $)$ BGAL1 is an abundant apoplastic protein. Proteins in total leaf extract (TE) and apoplastic fluids (AF) isolated from leaves of untreated $N$.

benthamiana plants were in-solution digested with trypsin and peptides were quantified by label-free quantification on $n=4$ biological replicates (6). Majority protein groups from $N$. benthamiana found in three of the four biological replicates of either AF or TE were retained. The $\log _{2}$ of the sum of the LFQ intensities is plotted against the distribution of LFQ intensities over the two proteomes $[\mathrm{AF} /(\mathrm{TE}+\mathrm{AF})]$. Protein groups annotated with GO:0003735 (ribosome) and GO:0016798 (glycosidases) are indicated in orange and blue, respectively. 


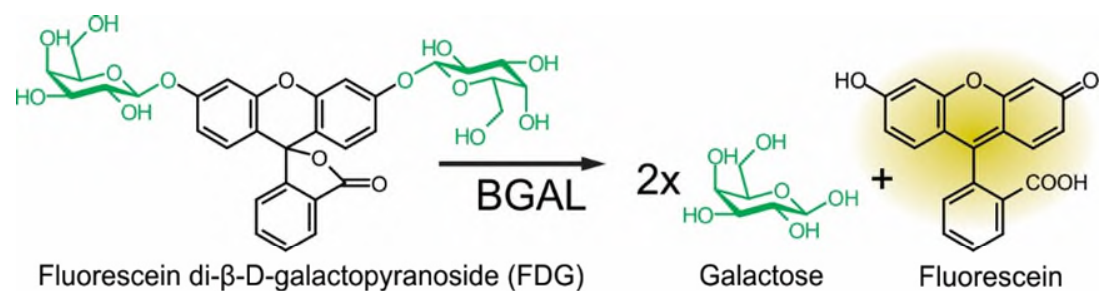

Figure S2 Assay for $\beta$-galactosidase activity.

Fluorescein-di- $\beta$-D-galactopyranoside (FDG) is cleaved into fluorescein and galactose. Fluorescein is detected by fluorescence at ex485/em535 $\mathrm{nm}$ using a fluorescence plate reader.

A

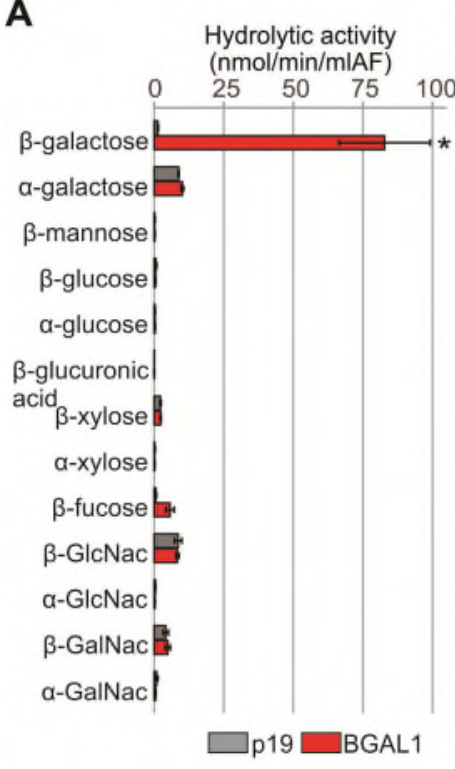

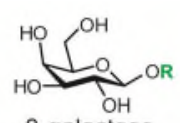

$\beta$-galactose
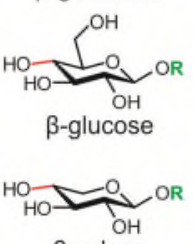

$$
\beta \text {-xylose }
$$

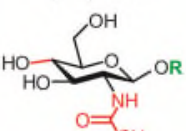

$\mathrm{O}=\mathrm{CH}$

$\beta-\mathrm{GlcNa}$

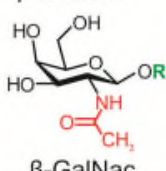

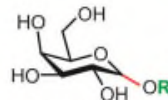

$\alpha$-galactose
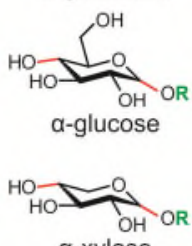

a-xylose

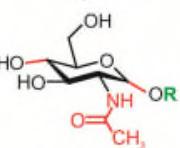

a-GlcNac

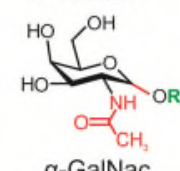

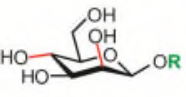

$\beta$-mannose

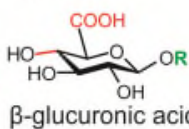

$\beta$-glucuronic acid

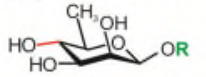

$\beta$-fucose

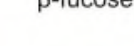

B

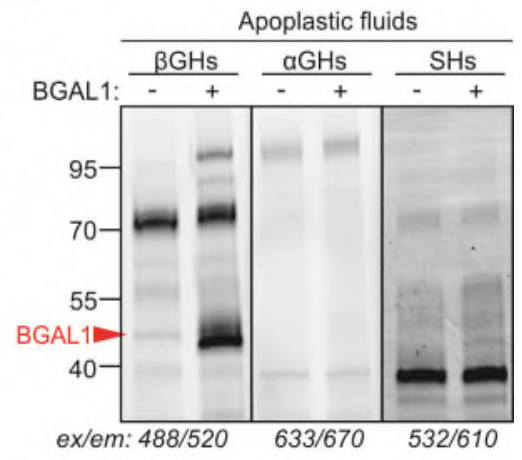

Figure S3 Further characterization of BGAL1 activity.

(A) BGAL1 hydrolyses 4-nitrophenyl-conjugates of $\beta$-galactose, but no other monosaccharide. Apoplastic fluids from agroinfiltrated plants transiently over expressing BGAL1 or p19 alone were incubated with $1 \mathrm{mM}$ 4-nitrophenyl conjugates at $\mathrm{pH} 5$ for $10 \mathrm{~min}$ at $\mathrm{RT}$. The reaction was stopped by adding $1 \mathrm{M} \mathrm{Na}_{2} \mathrm{CO}_{3}$ and the released 4-nitrophenol was measured at $420 \mathrm{~nm}$. Error bars, mean $\pm \mathrm{SE}$ from $\mathrm{n}=3$ replicates. Students $t$-test statistics $(* \mathrm{p}<0.05)$.

(B) BGAL1 is only labeled by JJB70. (A,B). Apoplastic fluids from agroinfiltrated plants transiently over expressing BGAL1 (+) or the non-accumulating mutant BGAL1 (-) control were incubated with 1 $\mu$ M JJB70 (labels $\beta$ Glycosyl Hydrolases, $\beta \mathrm{GHs}$ ), $2 \mu \mathrm{M} J J B 383$ (labels $\alpha \mathrm{GHs}$ ) and $0.2 \mu \mathrm{M}$ FP-Tamra (labels Ser hydrolases, SHs) at pH5 for $1 \mathrm{~h}$ at RT in the dark. Proteins were separated on protein gels and analysed by in-gel fluorescence scanning at different excitations (ex) and emissions (em) to detect the different fluorophores. 


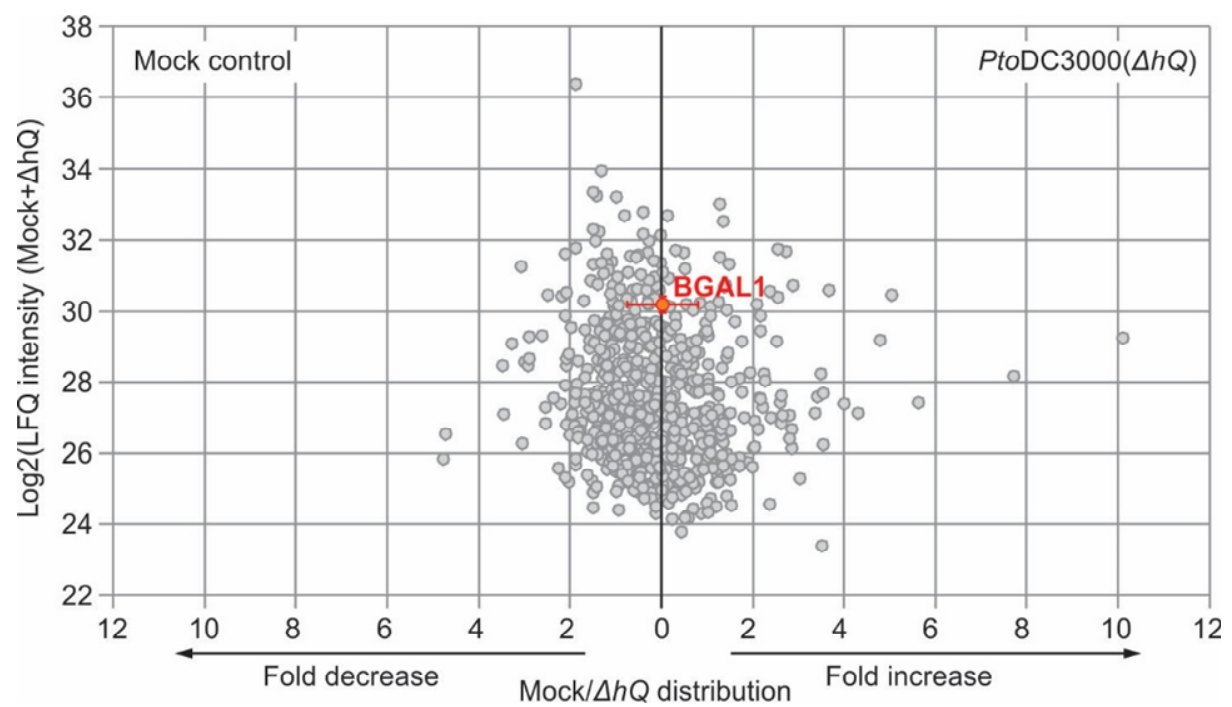

Figure S4 BGAL1 protein level is unaltered upon infection.

BGAL1 protein levels in apoplast are unaltered upon infection. AF from PtoDC3000( $\Delta h Q)$-inoculated plants and the mock control isolated at 2dpi were subjected to in-solution-digest with trypsin and proteins were quantified using label-free proteomics. Only proteins identified in all eight samples are shown. Error bars on BGAL1 (red) represent mean \pm SD of $n=4$ biological replicates: $\log _{2}(\mathrm{LFQ}$ intensity) $=30.20 \pm 0.20$ and Mock $/ \Delta h Q$ ratio $=0.02 \pm 0.76$-fold increase $(\mathrm{p}$-value $=0.96)$. 
A
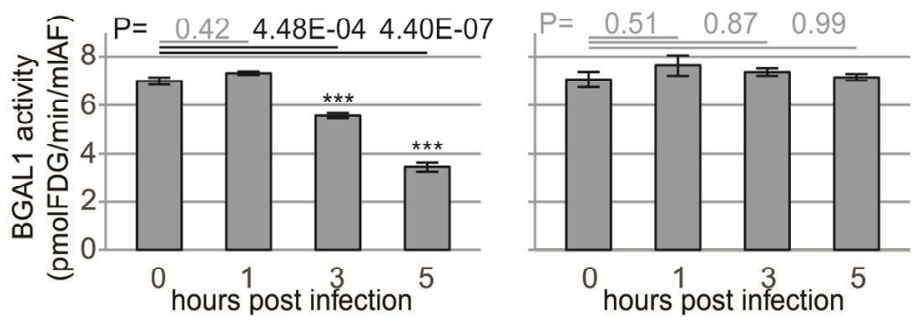

B
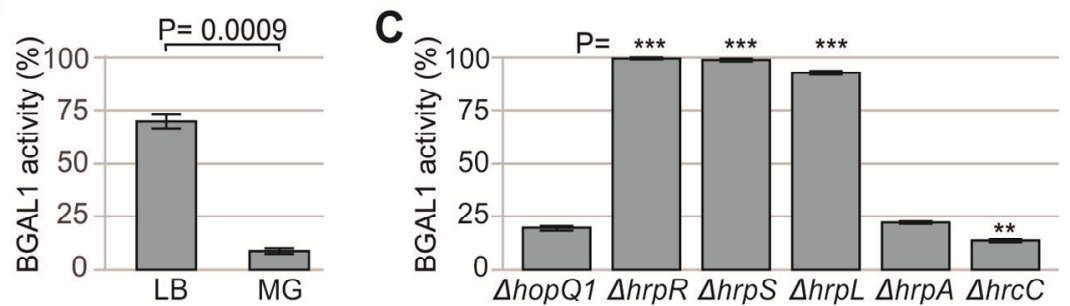

Figure S5 Characterization of BGAL1 inhibitor production in bacteria.

(A) BGAL inhibitor production upon infiltration is detected from 3 hours onwards. Water (Mock) or $10^{8} \mathrm{CFU} / \mathrm{ml}$ PtoDC3000 $\Delta h Q$ was infiltrated in $N$. benthamiana leaves and apoplastic fluids were isolated at $\mathrm{t}=0,1,3$ and 5 hours post infection. Apoplastic fluids were heated $\left(5^{\prime} 95^{\circ} \mathrm{C}\right)$ and tested for the BGAL1 inhibitor by incubating with the FDG substrate and BGAL1-containing apoplastic fluids from non-infected plants. Error bars, mean $\pm S E$ from $n=3$ replicates. Students $t$-test statistics p-values. (B) More BGAL1 inhibitor is produced by $\operatorname{PtoDC} 3000(\Delta h Q)$ when grown in virulence-inducing minimal (mannitol-glutamate (MG)) medium supplemented with iron, when compared to bacteria that are grown in rich (Luria-Bertani (LB)) medium. The bacteria were grown from $\mathrm{OD}_{600}=0.5$ for $24 \mathrm{~h}$. The supernatant of each culture was mixed with apoplastic fluid (AF) from $N$. benthamiana in an equal volume in the presence of $0.2 \mu \mathrm{M}$ FDG. Hydrolysis of FDG was measured using a fluorescence plate reader. Error bars, mean $\pm \mathrm{SE}$ of $\mathrm{n}=3$ replicates. $\mathrm{P}$ value was calculated using the $t$-test.

(C) The BGAL1 inhibitor is not produced by PtoDC3000 mutants lacking virulence regulators hrpR, $h r p S$, or $h r p L$, but is still produced by mutants lacking the T3SS, $h r p A$ and $h r p C$. This experiment was performed as in (A) in MG medium. Error bars, mean $\pm \mathrm{SE}$ from $n=3$ replicates. Tukey HSD test: **, $<0.001 ; * * *,<0.0001$. 

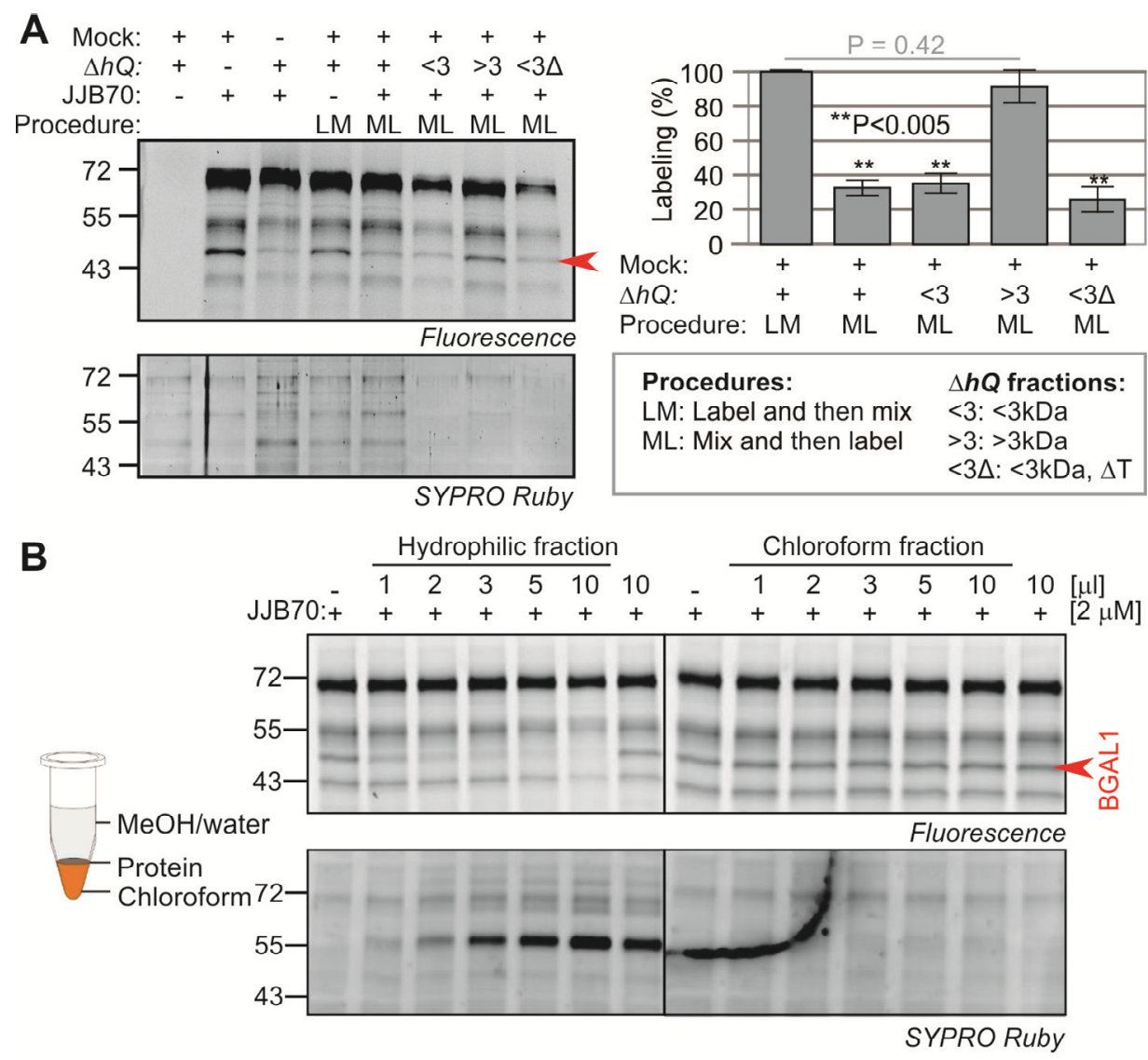

C

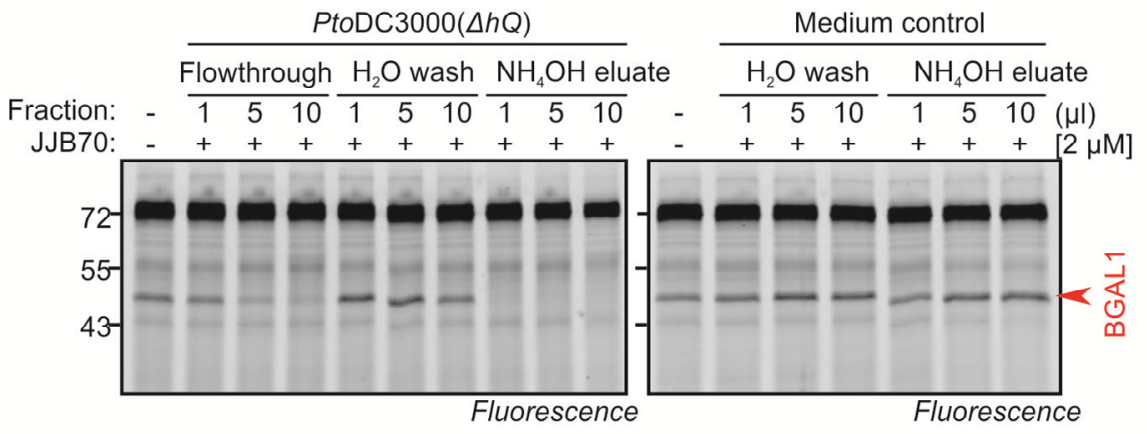

D

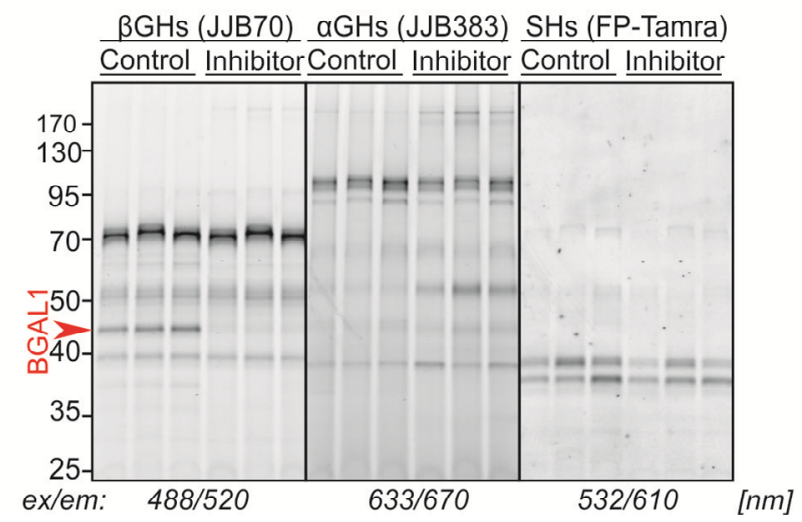

Figure S6 Further characterization of the BGAL1 inhibitor.

(A) BGAL1 inhibitor is a heat-stable small molecule. AFs from infected leaves isolated at 2 dpi were separated in $<3$ and $>3 \mathrm{kDa}$ fractions and the filtrate was heated at $95{ }^{\circ} \mathrm{C}$ for $5 \min (\Delta \mathrm{T})$. For mix-andlabel (ML), apoplastic fluids from infected leaves and the mock control were first mixed in a 1:1 ratio and then labeled with JJB70. For label-and-mix (LM), AFs of Mock and $\Delta h Q$ treated samples were separately labeled with JJB70 and then mixed in 1:1 ratio. The proteomes were separated on protein 
gels, detected by in-gel fluorescent scanning and the same gel was stained with SYPRO Ruby. Right: quantification of BGAL1 labeling, normalized to the LM sample and expressed as a percentage. Error bars, mean $\pm \mathrm{SE}$ of $\mathrm{n}=3$ independent experiments. $t$-test P-values.

(B) The BGAL1 inhibitor is a hydrophilic small molecule. PtoDC3000 $(\Delta h Q)$ bacteria were grown in minimal media and molecules were extracted with chloroform-methanol. Low molecular weight compounds $(<3 \mathrm{kDa})$ were removed from the upper hydrophilic (methanol/water) phase and the lower hydrophobic (chloroform) phase using ultrafiltration spin columns. The filtrate fractions were heated at $95^{\circ} \mathrm{C}$ for $5 \mathrm{~min}$, freeze dried, and dissolved in the original volume of water. Chloroform/methanol extracted growth medium, freeze-dried and dissolved in $10 \mu \mathrm{l}$ water served as a control. AFs of $N$. benthamiana were pre-incubated with the different fractions, labeled with JJB70, and analysed as in (C).

(C) The BGAL1 inhibitor is a basic molecule. A chloroform-methanol precipitation was performed with $40 \mathrm{ml}$ supernatant of PtoDC3000( $\Delta h Q)$ culture grown in mannitol-glutamate medium or only the medium as control. The freeze dried hydrophilic phase was dissolved in $50 \mathrm{ml}$ water, adjusted to pH3.5 and added to the prepared cation-exchange dowex50 resin column. The flow-through was collected (Fraction A) and the column was washed with water (Fraction B) and eluted using $2.5 \mathrm{M}$ ammonium hydroxide $\left(\mathrm{NH}_{4} \mathrm{OH}\right.$, Fraction $\left.\mathrm{C}\right)$. The freeze dried fractions $\mathrm{A}, \mathrm{B}$ and $\mathrm{C}$ were dissolved in water and used for competitive ABPP with apoplastic fluids isolated from $N$. benthamiana using JJB70.

(D) The purified inhibitor fraction suppresses labeling only of BGAL1 and not of other $\beta$-glycosyl hydrolases ( $\beta \mathrm{GHs})$; or $\alpha$-glycosyl hydrolases ( $\alpha \mathrm{GHs})$, and Ser hydrolases (SHs). Apoplastic fluids from three independent plants were preincubated with Fraction C obtained from PtoDC3000 $(\Delta h Q)$ culture or the medium control (described in A), and incubated with $1 \mu \mathrm{M} \mathrm{JJB} 70$ (labels $\beta \mathrm{GHs}$ ), $2 \mu \mathrm{M}$ JJB383 (labels $\alpha \mathrm{GHs}$ ) and $0.2 \mu \mathrm{M}$ FP-Tamra (labels SHs) at pH5 for $1 \mathrm{~h}$ at RT in the dark. Proteins were separated on protein gels and analysed by in-gel fluorescence scanning at different excitations (ex) and emissions (em) to detect the different fluorophores. 


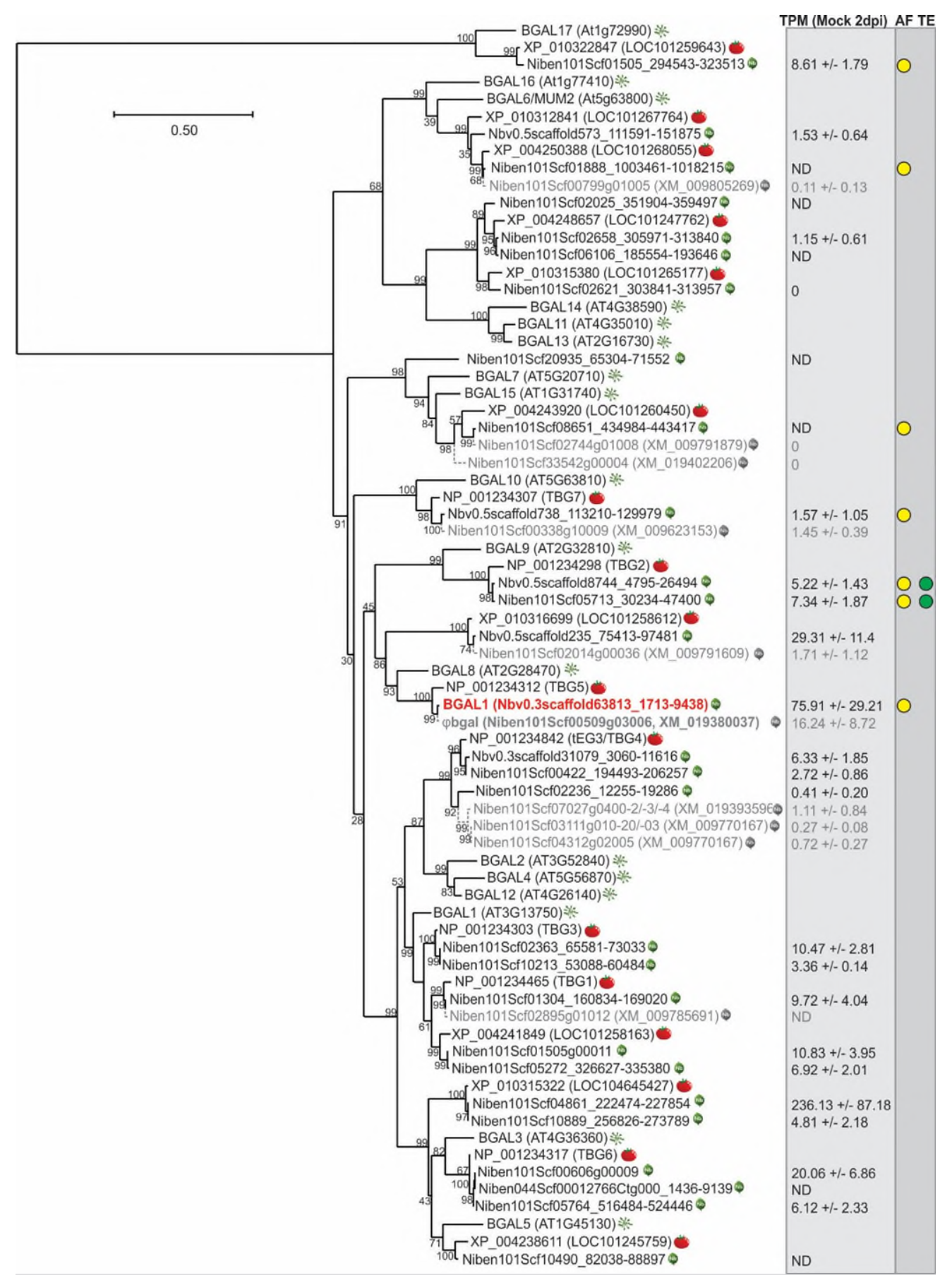

Figure $\mathbf{S 7}$ Molecular phylogenetic analysis of BGAL1 homologs.

72 sequences containing the GH35 domain (PF01301) were aligned with Clustal Omega and the residues corresponding to BGAL1 position 26-732 (lacking the SP and PF02140 domains) were extracted and re-aligned using Clustal Omega. The evolutionary history was inferred by using the Maximum Likelihood method and the tree with the highest log likelihood (-36048.75) is shown with $\%$ bootstrap values on the branches. The tree is drawn to scale, with branch lengths measured in the number of substitutions per site. Putative pseudogenes are indicated in grey. Proteins identified in either apoplastic fluid (AF) or a total extract (TE) are indicated (6). Transcripts per million (TPM) values of mock-infiltrated leaves at $2 \mathrm{dpi} \pm \mathrm{SD}$ of $\mathrm{n}=3$ replicates (35). ND, not detected, also not at other time points (7). 


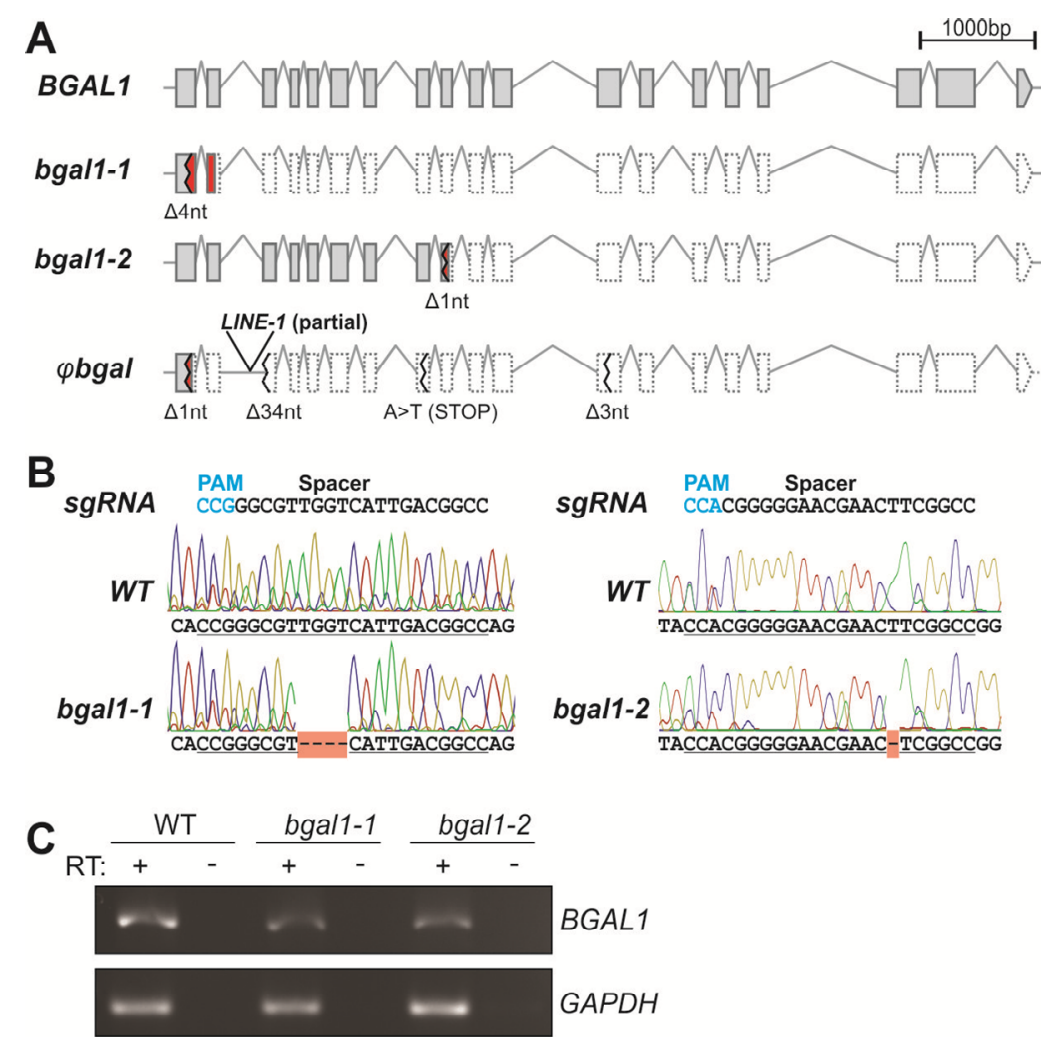

Figure S8 The two independent BGAL1 mutants grow and develop normally.

(A) Predicted exon-intron structure of BGAL1, both bgall mutants, and the $\varphi$ bgal pseudogene. Exons with correct in-frame translations (grey), non-translated exons (white) and CRISPR sites (wavy black lines) are indicated. The deletions cause a short out-of-frame translation until a stop codon (red). $\varphi$ bgal is a putative non-protein-encoding pseudogene containing a frame-shift mutation in exon 1 resulting in the introduction of a stop-codon after 4 non-synonymous amino acids (red). Additionally, a partial LINE1 retrotransposon insertion between exon 2 and 3 result in the truncation of exon 3 by 34 nucleotides. Finally, exon 8 contains a non-synonymous mutation introducing a stop-codon in the predicted correct frame. (B) Trace files of nucleotide sequences of PCR fragments amplified on the two CRISPR sites, showing the homozygous deletions in the bgall-1 and bgall-2 lines. (C) RT-PCR confirms unaltered transcript accumulation in both bgall mutants. RNA was isolated from leaves of untreated $N$. benthamiana plants and used as a template for RT-PCR with and without reverse transcriptase (RT). The BGAL1 amplification product spans the sgRNA target site. 

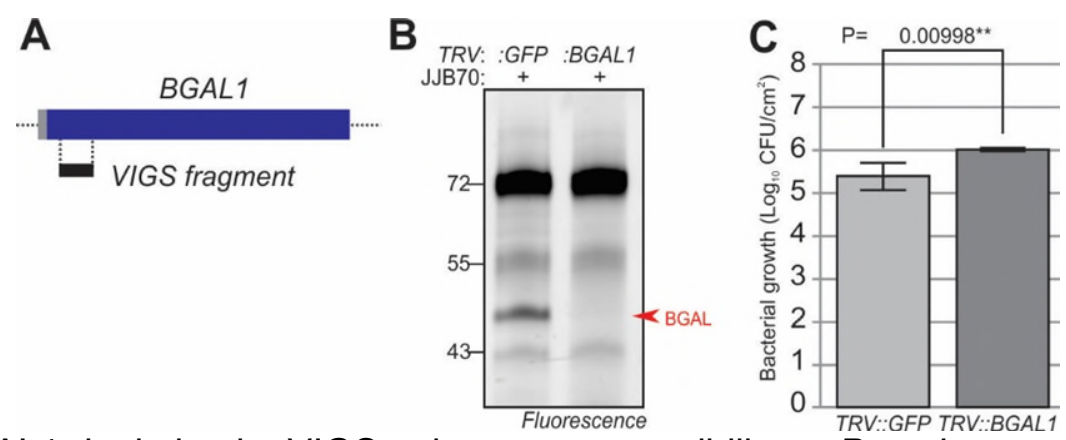

Figure S9 BGAL1 depletion by VIGS enhances susceptibility to $P$. syringae.

(A) Position of the fragment used for silencing, relative to the open-reading-frame encoding BGAL1 protein. (B) TRV::BGAL1 plants lack the JJB70 signal for BGAL1. Apoplastic fluid (AF) was isolated from $T R V:: G F P$ and $T R V:: B G A L 1$ plants 4 weeks after virus infection, and labeled with JJB70. Fluorescently labeled proteins were detected by fluorescence scanning. (C) TRV::BGAL1 plants are more susceptible to $P$. syringae. TRV::GFP and TRV::BGAL1 plants were spray-inoculated with $1 \times 10^{8}$ $\mathrm{CFU} / \mathrm{ml} P$ toDC3000 $(\Delta h Q)$ and bacterial growth was measured at 4 dpi. Error bars, mean $\pm \mathrm{SE}$ of $\mathrm{n}=3$ biological replicates.
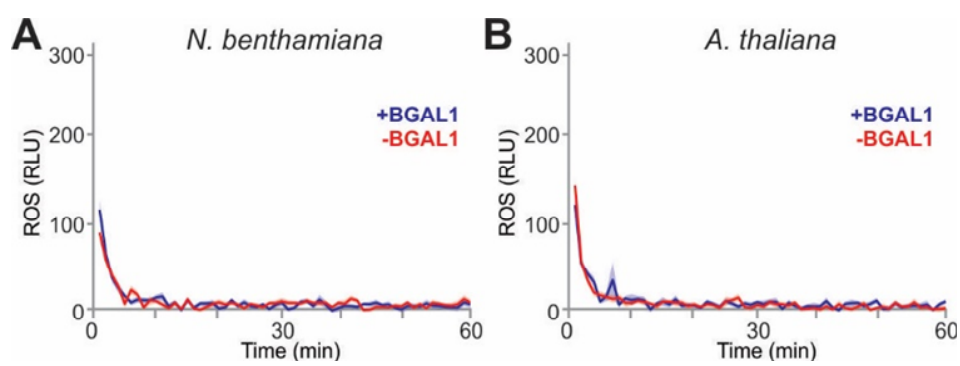

Figure S10 No ROS burst is triggered by apoplastic fluids in the absence of bacteria or flagellin.

Apoplastic fluids from $N$. benthamiana leaves overexpressing BGAL1 (+BGAL1) or mutant BGAL1 (-BGAL) as negative control, were added to leaf discs of $N$. benthamiana (A) or Arabidopsis (B) floating in horse radish peroxidase (HRP)/luminol-containing buffer. The ROS burst was monitored using a luminescence plate reader. Error intervals, mean $\pm S E$ for $n=12$ biological replicates.
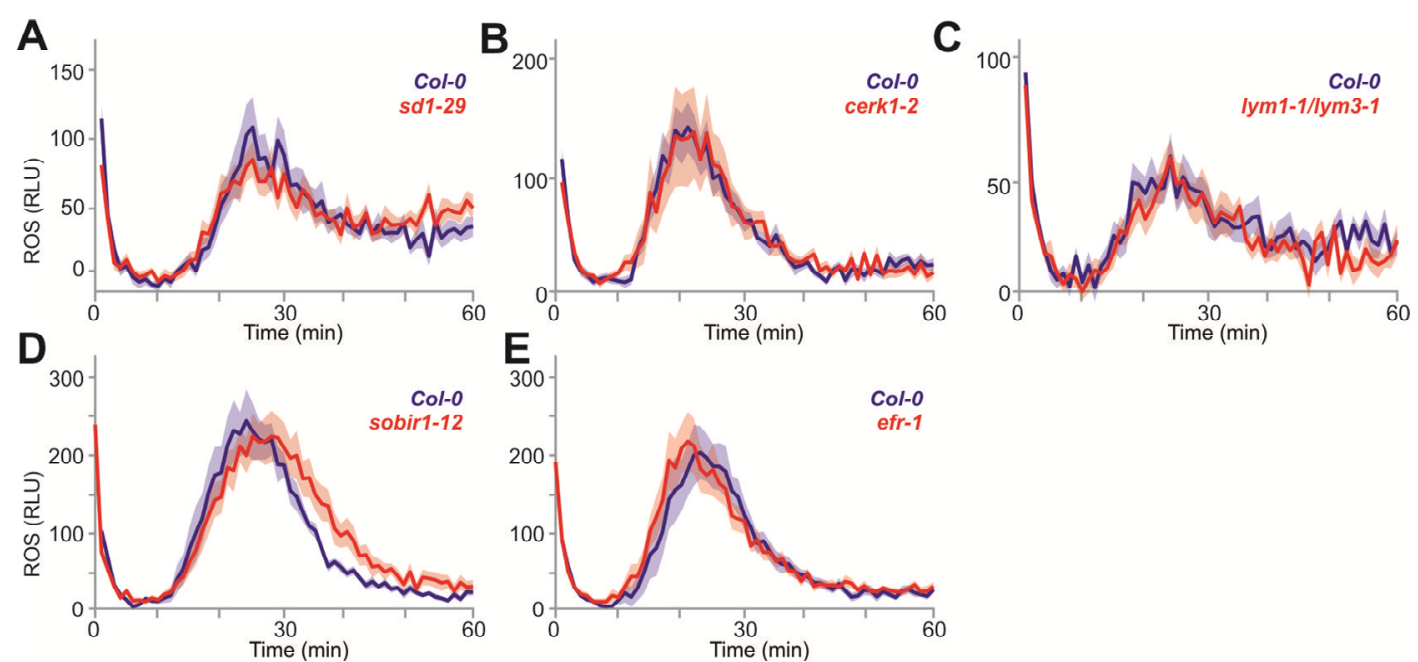

Figure S11 Arabidopsis sd1-29, cerk1, lym1/lym3,sobir1, and efr mutants still perceive the BGAL1-released elicitor.

PtoDC3000 $(\Delta h Q)$ bacteria were incubated with AF from $N$. benthamiana leaves overexpressing BGAL1. Treated bacterial solutions were added to leaf discs of mutant Arabidopsis floating in horse radish peroxidase (HRP)/luminol-containing buffer. The ROS burst was monitored in the Col-0 
control and in the sd1-29 (A), cerk1-2 (B), lym1-1/lym3-1 (C) sobirl-12 (D) and efr-1 (E) mutants using a luminescence plate reader. Error intervals, mean $\pm \mathrm{SE}$ for $\mathrm{n}=12$ biological replicates. RLU, relative luminescence units.

A

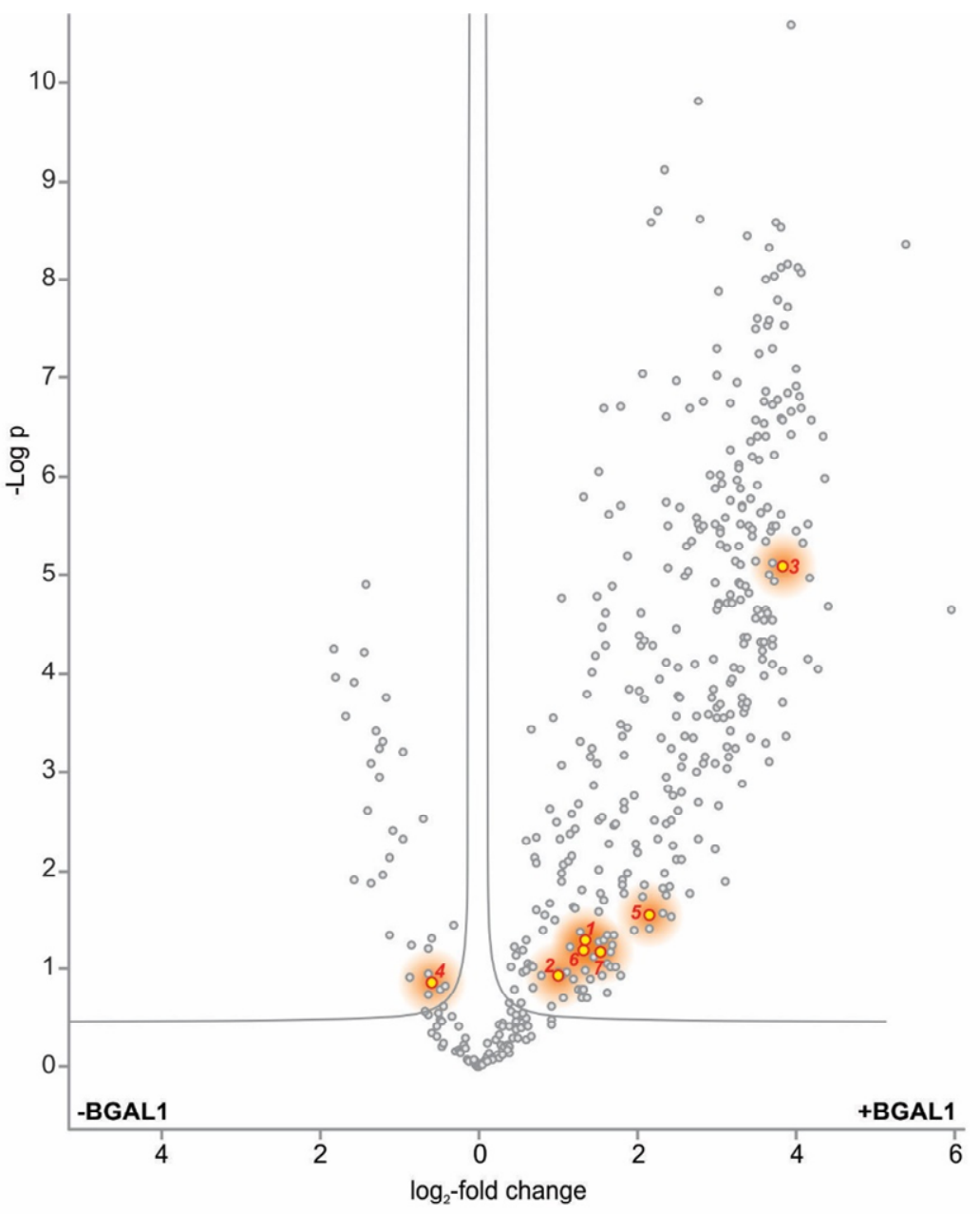

B

Pta6605 TRLSSGLKINSAKDDAAGLQIA flg22 QRLSTGSRINSAKDDAAGLQIA flg15 flg15- $\triangle 2$ RIKSAKDDAAGLQ

$\begin{array}{cc}\text { RIg15- } \Delta 2 & \text { RIKSAKDDAAGLQ } \\ 0 & \mathbf{1}\end{array}$ TRLSSGLKINSAKDDAAGLO LSSGLKINSAKDDAAGLO

SSGLKINSAKDDAAGLQ

SGLKINSAKDDAAGLQI SGLKINSAKDDAAGLQ KINSAKDDAAGLQ

$\log _{2}$ raw ion intensities (ND, not detected)

\begin{tabular}{|c|c|c|c|c|c|c|c|c|c|c|c|c|}
\hline \multicolumn{6}{|c|}{-BGAL } & \multicolumn{6}{|c|}{$+\mathrm{BGAL}$} & \multirow{2}{*}{$\begin{array}{c}\text { Peptide } \\
\text { score }\end{array}$} \\
\hline$\# 1$ & \#2 & \#3 & \#4 & \#5 & \#6 & \#1 & \#2 & \#3 & \#4 & \#5 & \#6 & \\
\hline 1.89 & 21.55 & 21.35 & 19.77 & ND & 20.92 & 22.10 & 22.28 & 23.04 & 21.65 & 21.51 & 21.30 & 93.85 \\
\hline 9.04 & 20.68 & 20.66 & 19.42 & 19.43 & 19.44 & ND & 21.73 & 22.43 & 20.67 & 20.54 & 20.47 & \\
\hline ND & ND & ND & ND & ND & ND & ND & 18.33 & 19.8 & 19.33 & 18.94 & 18.84 & \\
\hline 7.63 & 17.82 & 17.93 & 17.01 & 16.83 & 16.47 & ND & ND & ND & 17.82 & ND & ND & 129.88 \\
\hline 17.62 & 17.00 & 17.37 & ND & ND & ND & 17.36 & 17.71 & 17.91 & 17.84 & 17.56 & 17.48 & 117.65 \\
\hline 17.53 & 17.01 & 16.60 & ND & 16.73 & ND & 17.48 & 17.68 & 18.03 & 17.30 & 16.51 & 17.31 & 169.15 \\
\hline ND & 17.24 & ND & ND & 16.68 & 16.81 & 16.38 & 16.80 & 17.46 & 16.87 & 16.57 & 16.88 & 133.88 \\
\hline
\end{tabular}

Figure S12 Immunogenic peptides are released by apoplastic fluids containing BGAL1.

Purified flagellin of Pta6605 was incubated with apoplastic fluids isolated from $N$. benthamiana plants overexpressing BGAL1 (+BGAL1) or mutant BGAL1 (-BGAL1). Proteins were precipitated with $80 \%$ acetone and the peptide fraction (supernatant) was analyzed by MS for six technical replicates. All flagellin fragments are indicated and the flagellin peptides that contain the immunogenic region (KINSAKDDAAGLQ) are highlighted in the volcano plot (A) in red and summarized in (B). Similar results were obtained with a repetition experiment. 


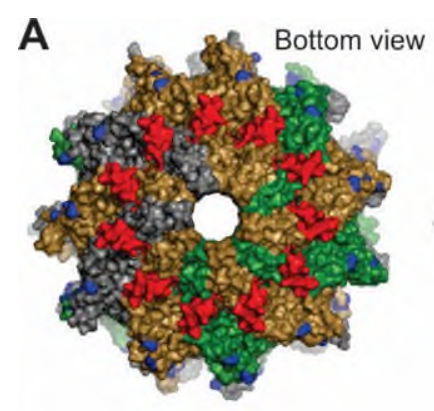

B

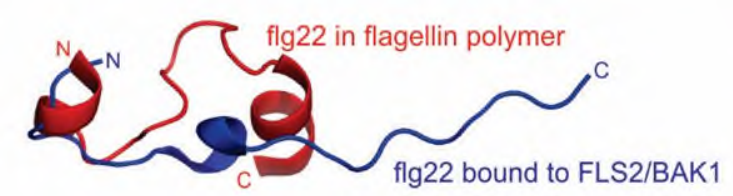

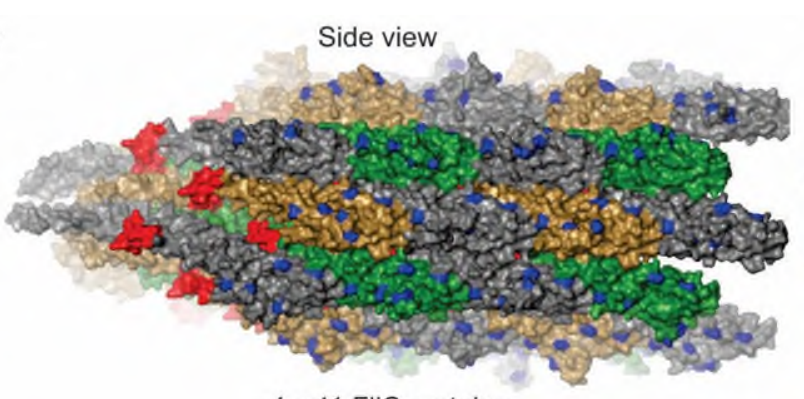
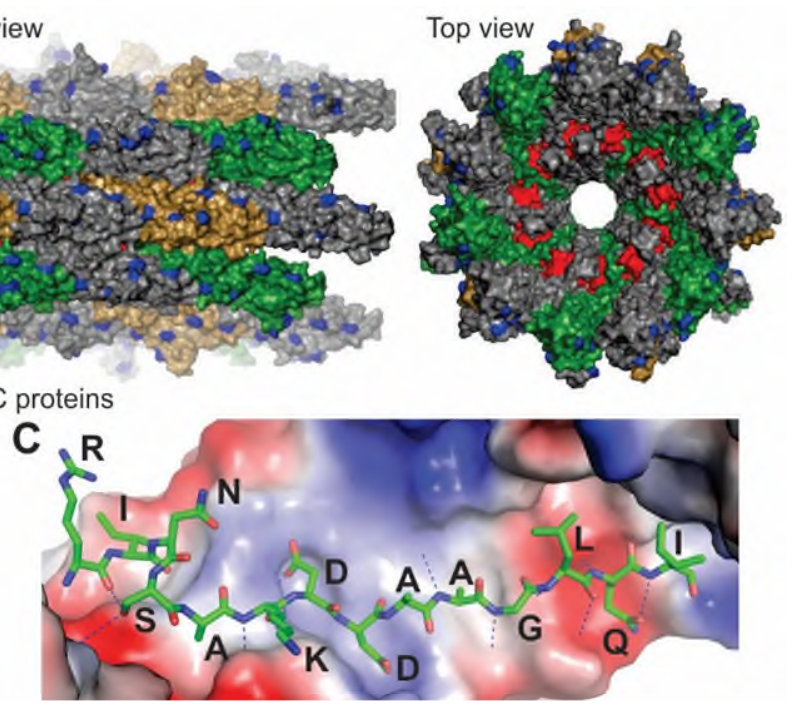

Figure S13 flg22 peptide is folded and buried inside the glycosylated flagellin polymer.

(A) Surface display of model of flagellar filament of PtoDC3000 modeled on P. aeruginosa (5wk6). Red, flg22; blue, $O$-glycosylation sites; grey/green/yellow, various flagellin subunits. (B) Structure of flg22 in FliC (red, modeled on 5wk6) and when bound to FLS2/BAK1 (blue, 4mn8). (C) Putative structure of flg15- $\Delta 2$ peptide (RINSAKDAAGLQI) bound to FLS2 showing its electrostatic surface charge (based on $5 \mathrm{wk} 6$ ).
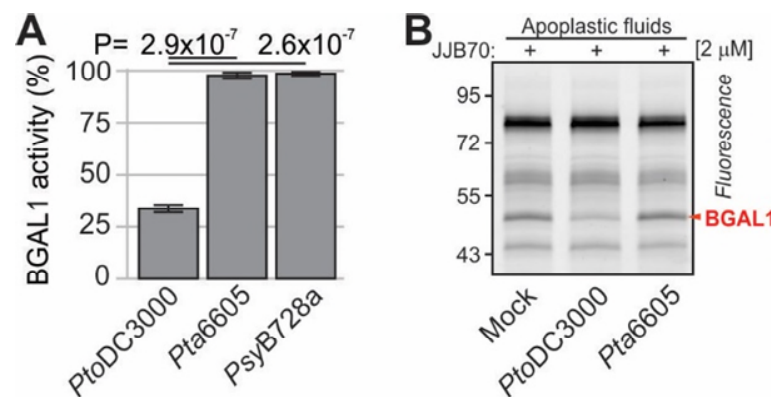

Figure S14 Pta6605 and PsyB728a do not produce a BGAL1 inhibitor.

(A) The bacteria were grown in M9 medium at $\mathrm{pH} 5.0$ supplemented with fructose from $\mathrm{OD}_{600}=0.5$ for $24 \mathrm{~h}$. The supernatant of each culture was mixed with an equal volume of apoplastic fluid (AF) from $N$. benthamiana in the presence of $0.2 \mu \mathrm{M}$ FDG. Hydrolysis of FDG was measured using a fluorescence plate reader. Error bars, mean \pm SE of $n=3$ replicates. Tukey HSD P values. (B) Selective BGAL1 suppression by PtoDC3000 but not Pta6605. Bacteria were grown in minimal media and metabolites were extracted using chloroform/methanol extraction. The $\mathrm{MeOH}$ fraction was freezedried, dissolved in the original volume of water and mixed 1:1 with apoplastic fluids from $N$.

benthamiana. JJB70 was added after 30 min and labeled proteins were detected from protein gels by fluorescence scanning. 

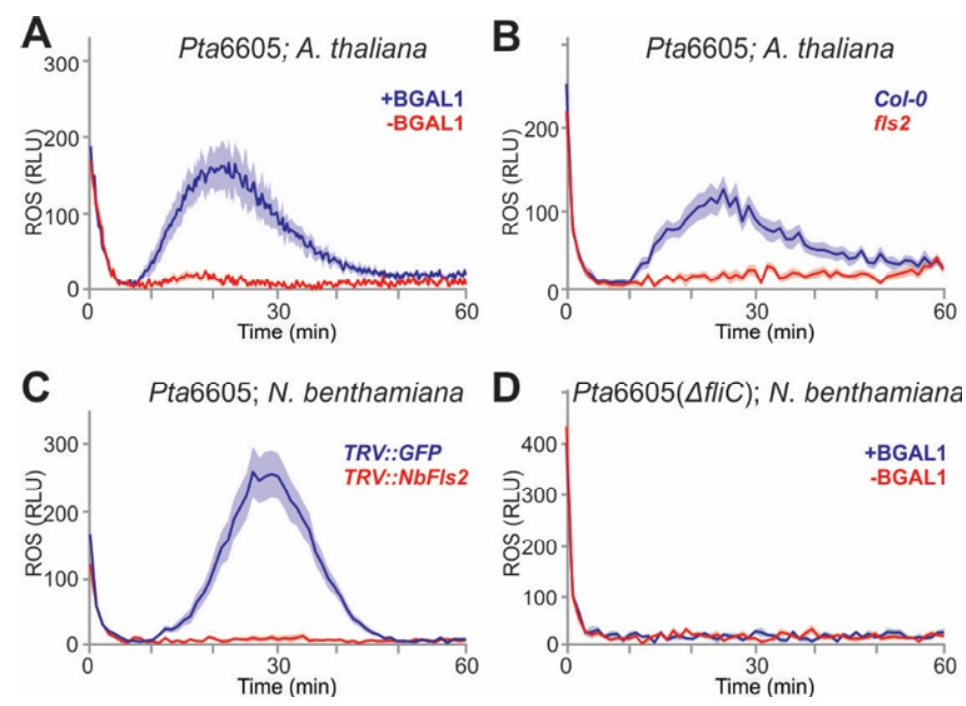

Figure S15 BGAL1 also releases the flagellin elicitor from Pta6605.

BGAL1 treated Pta6605 bacteria releases an elicitor that is detected by Arabidopsis leaf discs (A), but not by leaf discs of $f l s 2$ mutant Arabidopsis plants (B) or leaf discs from N. benthamiana silenced for NbFls2 (C), or when bacteria lacking flagellin were treated with BGAL1 (D). Pta6605 bacteria were incubated with AF from $N$. benthamiana leaves overexpressing BGAL1 (+BGAL1) or mutant BGAL1 as negative control (-BGAL1). Treated bacterial solutions were added to leaf discs of $N$. benthamiana plants floating in a horse radish peroxidase (HRP)/luminol-containing buffer. The ROS burst was monitored using a luminescence plate reader. Error intervals: mean \pm SE from n=6, 12, 40 and 6 biological replicates, respectively. RLU, relative luminescence units. 

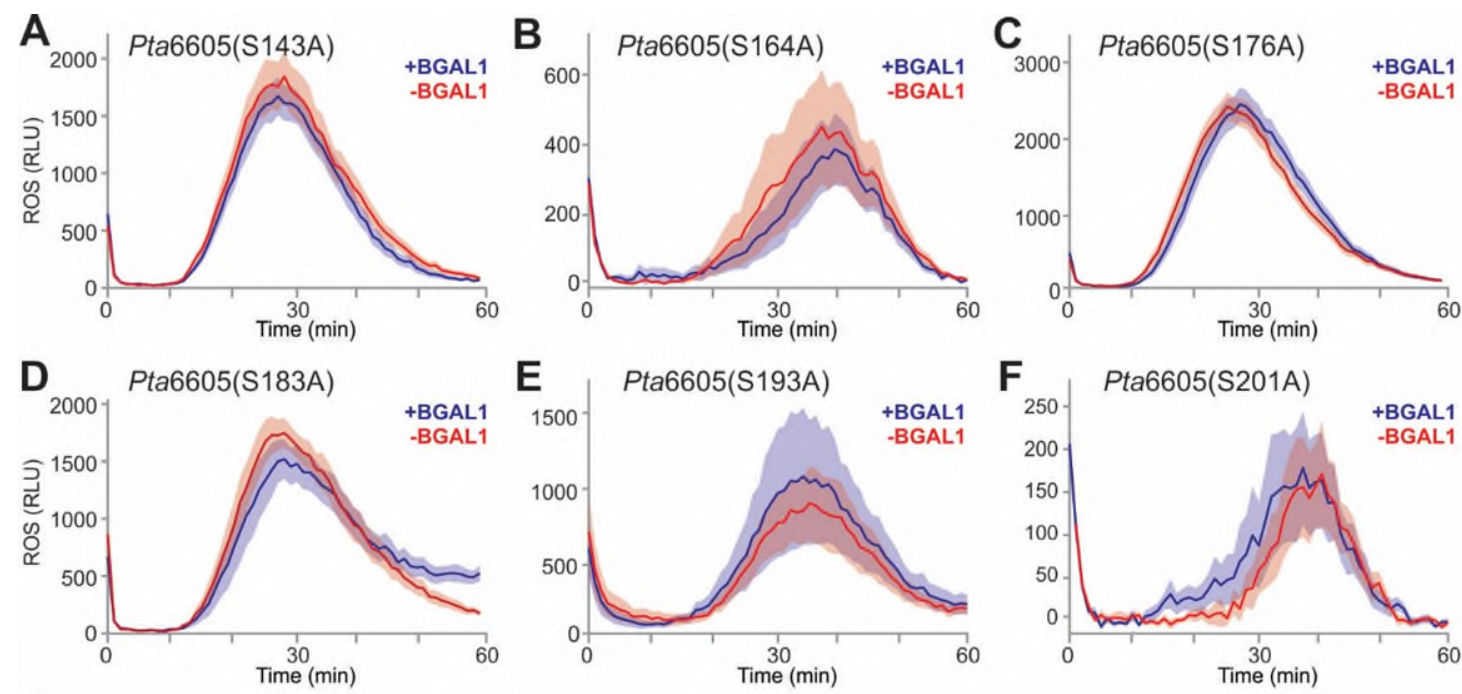

G

flg22 flg15- $\Delta 2$

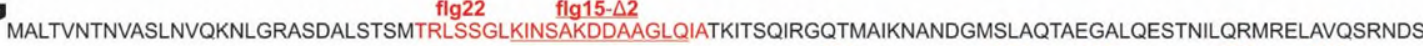
NSSTDRDALNKEFTAMSSELTRIAQSTNLNGKNLLDGSASTMTFQVGSNSGASNQITLTLSASFDANTLGVGSAVTIAGSDSTTAETNFSAAIAAIDSALQTINS TRADLGAAQNRLTSTISNLQNINENASAALGRVQDTDFAAETAQLTKQQTLQQASTSVLAQANQLPSAVLKLLQ

Figure S16 Single site glycan mutants trigger a BGAL1-independent ROS burst.

Mutant Pta6605 bacteria carrying substitutions S143A (A), S164A (B), S176A (C), S183A (D), S193A (E), or S201A (F) in flagellin, removing individual $O$-glycosylation sites, were incubated with apoplastic fluids from N. benthamiana leaves overexpressing BGAL1 (+BGAL1) or mutant BGAL1 (BGAL1). These samples were added to leaf discs of $N$. benthamiana floating in horse radish peroxidase (HRP)/luminol-containing buffer. The ROS burst was monitored using a luminescence plate reader. Error intervals: mean \pm SE from $n=12$ biological replicates. (G) Protein sequence of flagellin of Pta6605 highlighting the flg22 and flg15- $\Delta 2$ peptides (red) and the six O-glycosylation sites (blue).
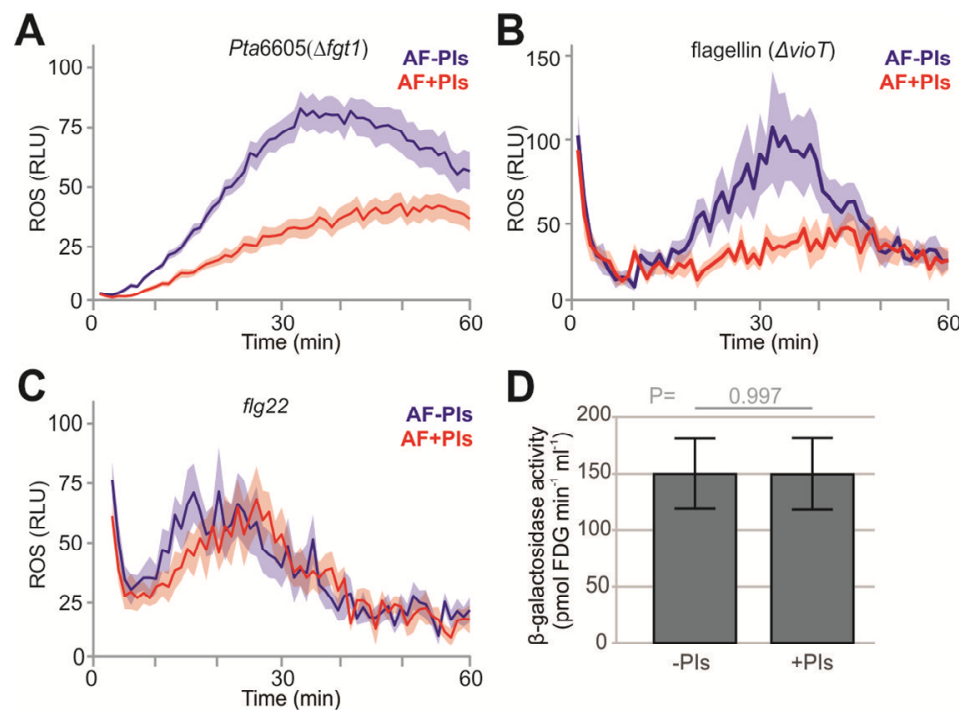

Figure S17 Protease inhibitor cocktail prevents elicitor release.

AF from $N$. benthamiana leaves overexpressing BGAL1 were pre-incubated with and without protease inhibitor cocktail and added to Pta6605( $\Delta f g t 1$ ) bacteria (A) of flagellin from Pta6601(4vioT) bacteria (B) or flg22 peptide (C). Treated samples were mixed with luminol and horse radish peroxidase (HRP), and added to leaf discs of $N$. benthamiana plants. The ROS burst was monitored using a luminescence plate reader. Error intervals, mean $\pm S E$ of $n=12(A, C)$ or $n=6(B)$ biological replicates. RLU, relative luminescence units. (D) Protease inhibitor cocktail does not affect BGAL1 activity. Apoplastic fluids were mixed with or without the protease inhibitor cocktail and $\beta$-galactosidase 
activity was measured using FDG as a substrate. Error bars: mean $\pm \mathrm{SE}$ of $n=4$ replicates. $t$-test Pvalue.
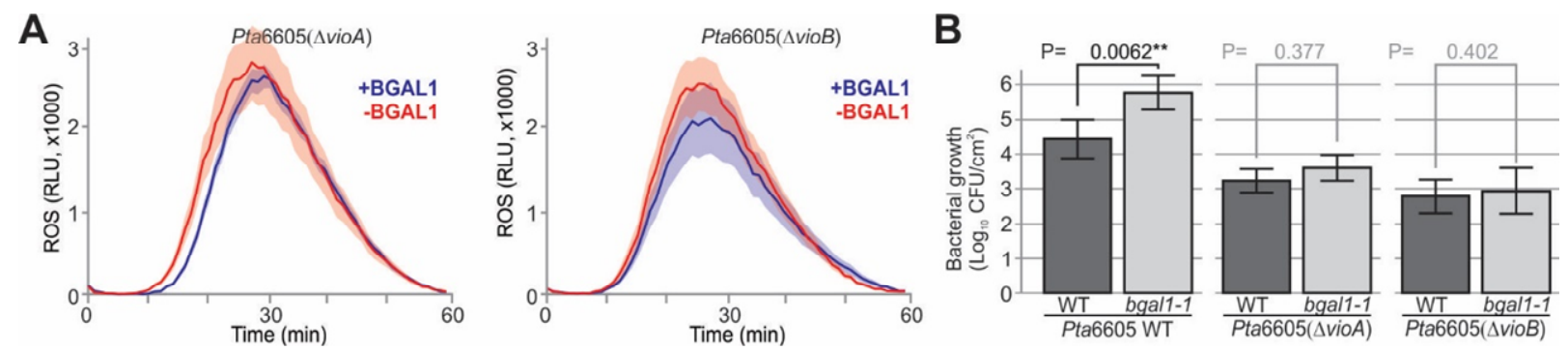

Figure S18 Pta6605 $\triangle v i o A$ and $\triangle v i o B$ mutants release elicitor irrespective of BGAL1 treatment. (A) Pta6605 $\Delta v i o A$ and $\triangle v i o B$ bacteria were treated with AF from $N$. benthamiana leaves overexpressing BGAL1 (+BGAL1) or mutant BGAL1 as negative control (-BGAL1). Treated bacterial solutions were added to leaf discs of $N$. benthamiana plants floating in a horse radish peroxidase (HRP)/luminol-containing buffer. The ROS burst was monitored using a luminescence plate reader. Error intervals, mean $\pm \mathrm{SE}$ from $\mathrm{n}=12$ biological replicates. (B) Bacterial growth of wildtype and mutant Pta6605 on WT and bgall-1 mutant plants at 3 dpi following spray inoculation with $1 \times 10^{8} \mathrm{CFU} / \mathrm{ml}$. Error bars, mean $\pm \mathrm{SE}$ of $\mathrm{n}=3$ biological replicates. RLU, relative luminescence units. 


\section{Methods}

Plants. Nicotiana benthamiana plants were grown in a growth chamber at $21^{\circ} \mathrm{C}$ and $\sim 60 \%$ relative humidity with a 12-h photoperiod and a light intensity of $2000 \mathrm{~cd} . \mathrm{sr}^{-\mathrm{m}^{-2}}$. Arabidopsis plants were grown in a Fitotron growth chamber (Weiss Gallenkamp, Loughborough, UK) at $21^{\circ} \mathrm{C}$ and $\sim 60 \%$ relative humidity with a $12 \mathrm{~h}$ photoperiod. Arabidopsis mutants used are all in Col-O background and are summarized in Table 1.

Table 1. Arabidopsis mutants lines used in this study

\begin{tabular}{|c|c|c|c|c|}
\hline Name & Locus & Origin & Reference & Provided by \\
\hline$s d 1-29$ & At1g61380 & SAIL_857_E06 & $(13)$ & NASC \\
\hline cerk1-2 & At3g21630 & GABI_096F09 & $(46)$ & A. Gust, Thübingen \\
\hline $\begin{array}{l}\text { lym1-1 } \\
\text { lym3-1 }\end{array}$ & $\begin{array}{l}\text { At1g21880/ } \\
\text { At1g77630 }\end{array}$ & $\begin{array}{l}\text { GABI_419G07 } \\
\text { SALK_111212 }\end{array}$ & (15) & A. Gust, Thübingen \\
\hline bakl-4 & At4g33430 & SALK_116202C & $(47)$ & NASC \\
\hline sobirl-12 & At2g31880 & SALK_050715 & $(48)$ & M. Joosten, Wageningen \\
\hline$f l s 2 c$ & At5g46330 & SAIL_691C4 & $(49)$ & C. Zipfel, Norwich \\
\hline
\end{tabular}

Bacterial strains. The P. syringae strains used in this study are listed in Table 2. PstDC3000 strains were grown in Luria-Bertani (LB) medium with $50 \mu \mathrm{g} / \mathrm{ml}$ rifampicin at $28^{\circ} \mathrm{C}$. Pta6605 strains were grown in King's $\mathrm{B}(\mathrm{KB})$ medium at $28^{\circ} \mathrm{C}$. For the infection assays, bacteria were cultured in LuriaBertani (LB) medium containing $10 \mathrm{mM} \mathrm{MgCl} 2$ at $28^{\circ} \mathrm{C}$.

Table 2. $P$. syringae strains used in this study

\begin{tabular}{|l|l|l|}
\hline Strain & Selection & Reference \\
\hline Pseudomonas syringae pv. tomato DC3000 $\Delta h Q$ & Rif & $(8)$ \\
\hline Pseudomonas syringae pv. tomato DC3000 $\Delta h Q \Delta f l i C$ & Rif & $(19)$ \\
\hline Pseudomonas syringae pv. tomato DC3000 $\Delta$ hrpR & Rif & $(50)$ \\
\hline Pseudomonas syringae pv. tomato DC3000 $\Delta h r p S$ & Rif & $(50)$ \\
\hline Pseudomonas syringae pv. tomato DC3000 $\Delta$ hrpL & Rif & $(50)$ \\
\hline Pseudomonas syringae pv. tomato DC3000 $\Delta h r p A$ & Rif & $(51)$ \\
\hline Pseudomonas syringae pv. tomato DC3000 $\Delta$ hrcC & Rif & $(51)$ \\
\hline Pseudomonas syringae pv. tabaci 6605 & - & $(52)$ \\
\hline Pseudomonas syringae pv. tabaci $6605 \Delta f l i C$ & - & $(52)$ \\
\hline Pseudomonas syringae pv. tabaci 6605 $\Delta$ fgtl & - & $(28)$ \\
\hline Pseudomonas syringae pv. tabaci $6605 \Delta v i o T$ & - & $(29)$ \\
\hline Pseudomonas syringae pv. tabaci 6605 $\Delta v i o A$ & - & $(29)$ \\
\hline Pseudomonas syringae pv. tabaci 6605 $\Delta v i o B$ & - & $(29)$ \\
\hline Pseudomonas syringae pv. tabaci 6605 S143A & - & $(28)$ \\
\hline Pseudomonas syringae pv. tabaci 6605 S164A & - & $(28)$ \\
\hline Pseudomonas syringae pv. tabaci 6605 S176A & - & $(28)$ \\
\hline Pseudomonas syringae pv. tabaci 6605 S183A & - & $(28)$ \\
\hline Pseudomonas syringae pv. tabaci 6605 S193A & - & $(28)$ \\
\hline Pseudomonas syringae pv. tabaci 6605 S201A & - & $(28)$ \\
\hline Pseudomonas syringae pv. syringae B728a & - & $(53)$ \\
\hline
\end{tabular}

Molecular cloning. All constructs were generated using standard molecular biology procedures. All new vectors are summarized in Table 3. All binary plasmids were transformed into Agrobacterium tumefaciens GV3101 (pMP90) by freeze-thawing and selected for kanamycin resistance.

The full length $B G A L 1$ open reading frame and its catalytic mutant $\left(B G A L 1^{\mathrm{E} 183 \mathrm{~A}, \mathrm{E} 254 \mathrm{~A}}\right)$ were commercially synthesized without the sequence encoding the signal peptide (SeqA, Biomatik, Cambridge, Ontario, Canada). The synthetic sequence (SeqA) contained flanking XhoI/SalI and BamHI restriction sites and a sequence encoding an $N$-terminal StrepII tag. The synthetic sequences were 
received in pBluescript (pBS) cloning vectors pBK23 and pBK25 for wild type and mutant BGAL1, respectively. The open reading frame was cloned into the pRH509 binary vector (54) using SalI and Bam $\mathrm{H} 1$ restriction sites, in between the $35 S$ promoter with a sequence encoding the PR1a signal peptide, and the PI-II terminator sites, resulting in binary vectors pBK26 (WT BGAL1) and pBK27 (mutant BGAL1), respectively.

The 281 bp fragment of BGAL1 (SeqB) used for Virus-induced Gene Silencing (VIGS) was commercially synthesized (Biomatik, Cambridge, Ontario, Canada) and was received in pBS vector pBK30. The fragment was cloned into binary TRV2 vector (55) using EcoR1 and BamH1 restriction sites resulting in binary vector $\mathrm{pBK} 46$.

The two CRISPR/Cas9 sgRNAs targeting BGAL were designed to minimize off-targets by using "CCTop - CRISPR/Cas9 target online predictor" (56) and their on-target activity was predicted according to (57). A CRISPR/Cas9 cloning vector (pL1V2-Kan-Cas9) was constructed by combining pAGM4723 (Addgene \#48015, (58)) with pICH47742 (Addgene \#49771, (59)), pICSL11017 (Addgene \#51144) and pICH47761 (Addgene \#48003, (58)) in a Golden Gate reaction with BpiI, resulting in pJK236. Specific PCR products were generated by amplifying from the template for sgRNAs pICH86966 (Addgene \#46966, (12)) using forward primers 5'ttggtctcaattggccgtcaatgaccaacgccgttttagagctagaaatagcaag-3' (for $\quad$ sgRNA_147/bgall-1) or 5'ttggtctcaattggccgaagttcgttcccceggtttagagctagaaatagcaag-3' (for sgRNA_106/bgall-2) and reverse primer 5'-ttggtctcaagcgtaatgccaactttgtac-3'. The two PCR products were combined with pJK236 and the AtU6 promoter (pICSL01009, Addgene \#46968, (12)) in a Golden Gate reaction with BsaI to generate pBK13 (sgRNA_106/bgal1-2) and pBK14 (sgRNA_147/bgall-1), respectively.

Table 3. Binary vectors generated in this study.

\begin{tabular}{|c|c|}
\hline Name & Description \\
\hline pBK26 & Binary vector carrying $35 S:: B G A L I(\mathbf{S e q} \mathbf{A})$ and $N p t I I$ on T-DNA \\
\hline pBK27 & Binary vector carrying $35 S: \because B G A L 1^{E 183 A, \mathrm{E} 254 \mathrm{~A}}$ and $N p t I I$ on T-DNA \\
\hline pBK13 & Binary vector carrying sgRNA_106, Cas 9 and NptII on T-DNA, used to generate bgall-2 \\
\hline pBK14 & Binary vector carrying $\boldsymbol{s} \boldsymbol{g} \boldsymbol{R N A} \_\mathbf{1 4 7}$, Cas 9 and $\mathbf{N p t I I}$ on T-DNA, used to generate bgall-1 \\
\hline pBK46 & Binary vector carrying TRV2 with 281 bp fragment (SeqB) of BGAL1 on T-DNA \\
\hline
\end{tabular}

SeqA: (synthetic BGAL1-encoding sequence) Bold underlined: codons encoding active site glutamates were mutated into $G C A$ and $G C G$ in the $B G A L 1^{\mathrm{E} 183 \mathrm{~A}, \mathrm{E} 254 \mathrm{~A}}$ catalytic mutant. Underlined: SeqB used for VIGS. Bold: restriction sites. Grey: two silent substitutions introduced to remove EcoRI sites. atgtcgacctcgagctggtctcatcct caatttgaaaagGCCAATGTGACGTATGATCACCGGGCGTTGGTCATTGACGGCCAGCGTAGAGTTC TCATCTCCGGCTCCATACATTACCCTCGTAGTACTCCTGATATGTGGCCAGACCTTATACAGAAATCTAAAGATGGAGGATTGGATGTACTAGA GACATATGTTTTCTGGAACATACATGAACCTGTTAGAAATCAGTATGATTTTGAAGGAAGGAAAGATTTGGTTAAATTTGTGAAGTTGGTGGGG AAAGCTGGCTTATATGCTCATATAAGGATCGGGCCTTATGTTTGTGCAGAATGGAACTATGGTGGGTTTCCTCTTTGGTTGCATTTCATTCCTG GAGTTGAATTTCGAACTGATAATGAACCATTCAAGGCAGAAATGAAGCGGTTTACAGCAAAAATTGTTGACATGCTCAAGCAAGAAAATCTTTA TGCATCCCAAGGGGGACCGGTTATTTTGTCTCAGATAGAAAATGAATATGGCAATGGGGATATTGAGTCTCGTTATGGTCCTCGTGCCAAACCT TATGTCAACTGGGCAGCAAAAATGGCTACATCGTTGGATACAGGAGTGCCATGGGTTATGTGCCAGCAACCAGATGCCCCTGATCCCATAATAA ACACTTGCAATGGATTCTATTGTGACCAATTCAAGCAGAACTCCGATAAGACACCCAAGATGTGGACGGAGAATTGGACTGGATGGTTTCTTTC TTTTGGTGGTGCTGTCCCTTACAGACCTGTGGAAGACATTGCTTTTTCTGTGGCTCGATTTTTCCAGCGAGGTGGAACCTTTCAGAACTATTAC ATGTACCACGGGGGAACGAACTTCGGCCGGACCAGTGGTGGGCCATTTATTTCAACTAGCTATGACTATGATGCTCCTTTAGATGAGTATGGCC TTATAAGACAACCAAAGTGGGGCCACTTGAAAGATCTCCATAAAGCCATAAAGCTGTGCGAGGCTGCAATGGTGGCAACTGATCCAACTATCAC TTCTCCAGGATCTAACATAGAGGTCAGTGTTTATAAAACTGGATCGGTGTGTGCTGCATTTCTTGCCAATGTGGGTACACAGTCTGACGCAGCC GTGACTTTCAATGGAAATTCATATCATTTGCCTCCTTGGTCCGTGAGCATCTTACCTGATTGCAAAAATGTGGCATTTAGTACTGCAAAGATTA ACTCGATGTCAACAATCTCAAAGTTTGTTACTCAGTCTACGGAAGCTGATGGTTCTGGCGCATCCTTGTCAGGTTGGACTTGGGTTAATGAGCC TGTAGGTATCTCAAGTGATAACGCGTTCACAAAAACGGGTTTGATGGAGCAGATAAATACTACAGCAGATAAAAGTGATTATCTTTGGTACTCT CTGAGTGTTAATGTAAAAAATGACGAGCCTTTCCTTCAAGATGGATCTCAAACAGTACTTCATGTGGAATCACTTGGCCATGTTCTTCATGCTT TCATTAACGGAAAGCTATCAGGAAGTGGGAAAGGAAACAGTGGAAATTCTAAAGTTACAATTGATGTTCCTGTCACCCTTGTACCTGGAGAAAA CAAAATCGACCTGTTGAGTGTGAATGTGGGGCTTCAGAACTATGGAGCATTCTTTGATCTTAAGGGAGCAGGTATTACCGGTCCTGTGCAATTG AAAGGCTTCAAAAATGGCTCTACTATTGATCTTTCGTCAAAGCAATGGACATATCAGGTTGGATTGAAAGGAGAAGAACTGGGGTTATCTGATG GAAGTTCTTCGCTTTGGAACTCACAATCTGCATTGCCTACAAACCAACCATTAATTTGGTATAAGGCAAGTTTTGATGCCCCTGCTGGAGATAC CCCTCTTTCACTAGATTTTACTGGAATGGGAAAGGGTGAAGCATGGGTGAATGGACAAAGCATTGGCCGATTTTGGCCTACCAATACTGCATCA AATGGAGGTTGTACTGACTCCTGCAATTATAGAGGATCTTACAATTCTAACAAATGTCTTAAAAATTGTGGAAAACCATCCCAGCTGCTATACC ACGTTCCTCGTTCATGGCTGCAATCCACGGGAAATGTCATAGTGTTGTTTGAGGAAATGGGTGGGAATCCAACAAAGCTATCTTTTGCAACAAG AGAGACAAGTAGTATATGCTCACGAGTTTCAGAGGCGCATCCACTTCCTATTGACAAGTGGACGTCGGATGACGATGCACGAAAGAAAGTAGGG CCAACTCTGTCTCTTGAGTGCCCTCGTCCTGATCAAGTCATTTCTTCAATCAAATTTGCAAGCTTTGGCACTCCTCTTGGTGCATGTGGAAGCT TTAGCCATGGTCGATGCACAAGCAACAATGCTCTTTCCCATGTAAAGAAGGCTTGCATTGGATCGAGACACTGTAGTGTTGGAGTTTCAATAGA TGTATTTGGTGATCCATGTATAGGAGTCACAAAAAGTTTAGCCGTAGAAGCTTCCTGTTCGggatcc-3'

SeqB: fragments used for silencing BGAL1. Bold: restriction sites.

gaattcCTGTTAGAAATCAGTATGATTTTGAAGGAAGGAAAGATTTGGTTAAATTTGTGAAGTTGGTGGGGAAAGCTGGCTTATATGCTCATAT AAGGATCGGGCCTTATGTTTGTGCAGAATGGAACTATGGTGGGTTTCCTCTTTGGTTGCATTTCATTCCTGGAGTTGAATTTCGAACTGATAAT 
Plant transformation. Agrobacterium mediated transformation of $N$. benthamiana was performed as described (60). Briefly, 4-week old N. benthamiana plants were agroinfiltrated with A. tumefaciens strain GV3101 pMP90 carrying binary plasmids pBK13 (carrying sgRNA_106 for bgal1-2) and pBK14 (carrying sgRNA_147 for bgall-1). At three days post-infiltration, the infiltrated leaves were cut into 1 $\mathrm{cm}^{2}$ sections and were cultured under sterile conditions on shooting media [per liter: 4.3gr MS basal salts (1x), $30 \mathrm{gr}$ sucrose, 1x B5 vitamins, $0.1 \mathrm{mg}$ Naphthalene Acetic Acid (NAA), 0.59 gr MES, pH 5.7 using $\mathrm{KOH}, 4$ gr Agargel, after autoclaving add 1mg Benzylaminopurine (BAP), 300mg Ticarcillin/clavulanic acid] for three days, and subsequently transferred to shooting media supplemented with $150 \mu \mathrm{g} / \mathrm{ml}$ kanamycin and cultured for approximately 2-3 weeks to select for stable transformants. Shoots were excised and transferred to rooting media [per liter: 2.15 gr MS basal salts $(0.5 \mathrm{x}), 5 \mathrm{gr}$ sucrose, $0.1 \mathrm{mg}$ NAA stock, pH 5.8 with $\mathrm{KOH}, 2.5$ gr Gelrite, after autoclaving add $300 \mathrm{mg}$ Ticarcillin/clavulanic acid] supplemented with $150 \mu \mathrm{g} / \mathrm{ml}$ kanamycin and were further cultured until roots appeared, after which plantlets were transferred to soil and grown in the growth chamber. To identify stable $N$. benthamiana transformants carrying mutant BGAL1 alleles, the targeted segment of the BGAL1 gene was PCR amplified using primers for bgall-1 (forward primer: 5' - tctgcttctaagaacagat3' and reverse primer 5'-gtctctagtacatccaatcc-3') and bgall-2 (forward primer: 5'accatactcatctaaaggagca-3' and reverse primer: 5'-ggctcgattttccagcgag-3') and the PCR fragments were sequenced by sanger sequencing to confirm mutations. Mutant lines were screened by ABPP using the JJB70 probe to confirm BGAL1 depletion in the T2 and T3 generations.

Virus-induced Gene Silencing (VIGS). Agrobacterium cultures were grown overnight and resuspended in agroinfiltration buffer $\left(10 \mathrm{mM} \mathrm{MgCl}_{2}, 10 \mathrm{mM}\right.$ MES pH 5.0, and $100 \mu \mathrm{M}$ acetosyringone $)$ at $\mathrm{OD}_{600}=0.5$. Bacteria containing $\mathrm{pBK} 46(T R V: \because B G A L 1)$ or $T R V 2: \because G F P$ were mixed $1: 1$ with bacteria containing TRV1 (55). After incubation for $1 \mathrm{~h}$ at room temperature, the mixed cultures were infiltrated into leaves of 10-day-old $N$. benthamiana plants. The infiltrated seedlings were grown for 3-4 weeks in a growth chamber until use.

Agroinfiltation. For transient expression of proteins in $N$. benthamiana, overnight cultures of Agrobacterium carrying binary vectors were harvested by centrifugation. Cells were resuspended in induction buffer (10 mM MgCl $2,10 \mathrm{mM}$ MES pH5.0, and $100 \mu \mathrm{M}$ acetosyringone) at $\mathrm{OD}_{600} 0.5$. After $1 \mathrm{hr}$ at $21^{\circ} \mathrm{C}$, cells were infiltrated into leaves of 4-week-old $N$. benthamiana. Leaves were harvested and processed at four days after agroinfiltration.

Isolation of apoplastic fluids. Apoplastic fluids were collected as described previously (61). Leaves from infected or agroinfiltrated plants were submerged in ice-cold water and infiltrated by applying vacuum for $5 \mathrm{~min}$. The surface of water-infiltrated leaves were dried with absorbing paper and leaves were carefully mounted in an empty $20 \mathrm{~mL}$ syringe, placed in a $50 \mathrm{ml}$ tube. Apoplastic fluids were collected by centrifugation at $2,000 \mathrm{~g}$ at $4^{\circ} \mathrm{C}$ for $20 \mathrm{~min}$ and used immediately.

In-solution digestion of apoplastic fluids for proteome analysis. $500 \mu 1$ of apoplastic fluids from $N$. benthamiana were isolated from 16 leaf discs. Proteins were reduced by incubation with dithiothreitol (DTT, final concentration $5 \mathrm{mM}$ ) for $30 \mathrm{~min}$ at room temperature. Samples were then alkylated with iodoacetamide (IAA, final concentration $20 \mathrm{mM}$ ) for $30 \mathrm{~min}$ at room temperature. Then the apoplastic fluids were chloroform-methanol precipitated and the precipitated proteins were dissolved in $100 \mu \mathrm{L}$ $6 \mathrm{M}$ urea. $500 \mu \mathrm{L}$ water was added and protein digestion was performed by adding $400 \mathrm{ng}$ trypsin/LysC mix (Promega, Madison, WI, USA). Sample was incubated at $37^{\circ} \mathrm{C}$ for $18 \mathrm{~h}$. The protein digestion was 
stopped by adding formic acid (FA, final $1 \% \mathrm{v} / \mathrm{v}$ ) and peptides were purified using the sep-paK C18 columns (Waters, Elstree, UK).

Proteome analysis of apoplastic fluids. Sample clean-up for LC-MS. Trypsin and LysC digested samples were desalted on home-made C18 StageTips as described (62). On each 2 disc StageTip around $15 \mu \mathrm{g}$ peptides were loaded (based on the initial protein concentration). After elution from the StageTips, samples were dried using a vacuum concentrator (Eppendorf) and the peptides were taken up into 10 $\mu \mathrm{L} 0.1 \%$ formic acid solution. Samples for the analysis endogenously digested peptides in the AF were generated by supplementing the AF with 4 volumes of MS-grade acetone, followed by incubation on ice for $1 \mathrm{~h}$ and centrifugation at $18,000 \mathrm{~g}$ for $15 \mathrm{~min} .4 / 5^{\text {th }}$ of the supernatants were then transferred to fresh Eppendorf tubes and the acetone evaporated by vacuum centrifugation. The dried peptide samples were then dissolved in $0.1 \%$ formic acid and immediately analysed without further clean-up.

$L C-M S / M S$. Experiments were performed on an Orbitrap Elite instrument (Thermo Fisher Scientific, Waltham, MA, USA, (63)) that was coupled to an EASY-nLC 1000 liquid chromatography (LC) system (Thermo Fisher Scientific, Waltham, MA, USA). The LC was operated in the one-column mode. For full AF analysis the analytical column was a fused silica capillary $(75 \mu \mathrm{m} \times 25 \mathrm{~cm})$ with an integrated PicoFrit emitter (New Objective) packed in-house with Reprosil-Pur 120 C18-AQ $3 \mu \mathrm{m}$ resin (Dr. Maisch). For analysis of endogenously digested peptides from the AF the analytical column had a length of $35 \mathrm{~cm}$ and the resin was Reprosil-Pur $120 \mathrm{C} 18$-AQ $1.9 \mu \mathrm{m}$ resin (Dr. Maisch). The analytical column was encased by a column oven (Sonation) and attached to a nanospray flex ion source (Thermo Fisher Scientific, Waltham, MA, USA). The column oven temperature was adjusted to $45^{\circ} \mathrm{C}$ during data acquisition. The LC was equipped with two mobile phases: solvent A $(0.1 \%$ formic acid, FA, in water) and solvent B $(0.1 \%$ FA in acetonitrile, ACN). All solvents were of UHPLC (ultra high performance liquid chromatography) grade (Sigma-Aldrich, Saint Louis, MO, USA). Peptides were directly loaded onto the analytical column with a maximum flow rate that would not exceed the set pressure limit of 980 bar (usually around $0.5-0.8 \mu \mathrm{L} / \mathrm{min}$ ). Peptides were subsequently separated on the analytical column by running a 70 or 140 min gradient of solvent A and solvent B (start with 7\% B; gradient $7 \%$ to 35\% B for 60 (or $120 \mathrm{~min}$ ); gradient 35\% to $100 \%$ B for $5 \mathrm{~min}$ (or $10 \mathrm{~min}$ ) and $100 \% \mathrm{~B}$ for $5 \mathrm{~min}$ (or $10 \mathrm{~min}$ )) at a flow rate of $300 \mathrm{nl} / \mathrm{min}$. The mass spectrometer was operated using Xcalibur software (version $2.2 \mathrm{SP} 1.48$ ). The mass spectrometer was set in the positive ion mode. Precursor ion scanning was performed in the Orbitrap analyzer (FTMS; Fourier Transform Mass Spectrometry) in the scan range of $\mathrm{m} / \mathrm{z} 350-1800$ and at a resolution of 60000 with the internal lock mass option turned on (lock mass was $445.120025 \mathrm{~m} / \mathrm{z}$, polysiloxane, (64)). Product ion spectra were recorded in a data dependent fashion in the ion trap (ITMS; Ion Trap Mass Spectrometry) in a variable scan range and at a rapid scan rate. The ionization potential (spray voltage) was set to $1.8-2.0 \mathrm{kV}$. Peptides were analyzed using a repeating cycle consisting of a full precursor ion scan $\left(1.0 \times 10^{6}\right.$ ions or $\left.30 \mathrm{~ms}\right)$ followed by 10 product ion scans $\left(1.0 \times 10^{4}\right.$ ions or $\left.50 \mathrm{~ms}\right)$ where peptides are isolated based on their intensity in the full survey scan (threshold of 500 counts) for tandem mass spectrum (MS2) generation that permits peptide sequencing and identification. CID (collision-induced dissociation) collision energy was set to $35 \%$ for the generation of MS2 spectra. During MS2 data acquisition dynamic ion exclusion was set to 60 seconds with a maximum list of excluded ions consisting of 500 members and a repeat count of one. Ion injection time prediction, preview mode for the FTMS, monoisotopic precursor selection and charge state screening were enabled. Only charge states $>1$ were considered for fragmentation.

Peptide and Protein Identification using MaxQuant. RAW spectra were submitted to an Andromeda (65) search in MaxQuant (version 1.5.3.30). For the analysis of trypsin/LysC digested samples we used the following settings (66): Label-free quantification and match-between-runs was activated (67). MS/MS spectra data were searched against the ACE_0319_Niben_Final.fasta $(N$. benthamiana, 42853 entries, (6)) and Uniprot reference database UP000002515_223283.fasta (Pseudomonas syringae pv. tomato (strain ATCC BAA-871 / DC3000), 5431 entries) databases. All searches included also a contaminants database (as implemented in MaxQuant, 267 sequences). The contaminants database contains known MS contaminants and was included to estimate the level of contamination. Andromeda searches allowed oxidation of methionine residues (16 Da), acetylation of protein $\mathrm{N}$-terminus (42 Da as dynamic modification and the static modification of cysteine (57 Da, alkylation with IAM). Enzyme specificity was set to "Trypsin/P" with 2 missed cleavages allowed. The instrument type in Andromeda searches was set to Orbitrap and the precursor mass tolerance was set to 
$\pm 20 \mathrm{ppm}$ (first search) and $\pm 4.5 \mathrm{ppm}$ (main search). The MS/MS match tolerance was set to $\pm 0.5 \mathrm{Da}$. The peptide spectrum match FDR and the protein FDR were set to 0.01 (based on target-decoy approach). Minimum peptide length was 7 amino acids. For protein quantification unique and razor peptides were allowed. Modified peptides were allowed for quantification. The minimum score for modified peptides was 40 .

For the analysis of endogenously digested peptides in the AF we used the following settings: Label-free quantification and match-between-runs was activated (67). MS/MS spectra data were searched against the p19_vector_proteins.fasta (2 entries) and ACE_0383_SOI_v02.fasta (containing the sequence of flagellin and BGAL1). As above, all searches included also a contaminants database (as implemented in MaxQuant, 267 sequences). Andromeda searches allowed oxidation of methionine residues (16 Da) and acetylation of protein N-terminus (42 Da) as dynamic modification. Enzyme specificity was set to "unspecific". The instrument type in Andromeda searches was set to Orbitrap and the precursor mass tolerance was set to $\pm 20 \mathrm{ppm}$ (first search) and $\pm 4.5 \mathrm{ppm}$ (main search). The MS/MS match tolerance was set to $\pm 0.5 \mathrm{Da}$. The peptide spectrum match FDR and the protein FDR were set to 0.01 (based on target-decoy approach). Minimum peptide length was 8 amino acids and the maximum length 25. For protein quantification unique and razor peptides were allowed. Modified peptides were allowed for quantification. The minimum score for modified peptides was 40 .

Data Analysis. Initial data analysis and filtering and statistical evaluation was performed by using the PERSEUS computational platform (version 1.5.5.3, (68)). For data generated from trypsin/LysC digested samples we performed the following manipulations: Briefly, only protein groups with at least two identified unique peptides over all runs were considered for further analysis. Non- $N$. benthamiana proteins and hits to the decoy database were removed. For quantification we combined related biological replicates into categorical groups and investigated only those proteins that were found in all samples. Comparison of protein group quantities (relative quantification) between different MS runs is based solely on the LFQ's as calculated by MaxQuant (MaxLFQ algorithm, (67)). For the statistics, each biological replicate was used for a pairwise comparison. The identified proteins were plotted by the mean of the sum of $\log _{2}$ (LFQ intensity) of the Mock and $\Delta h Q$ samples against the mean of ratio(Mock $/ \Delta h Q$ ) of four biological replicates. For the analysis of data generated from endogenously digested AF samples we analysed the peptide.txt output from MaxQuant. Peptide intensities were loaded into PERSEUS grouped into categorical groups (no_bGal or bGal) based on their treatment and normalized by subtraction from median. Missing values were then imputed and the categorical groups compared by performing a Student's $t$-test (FDR 0.05; S0 0.1; number of randomizations 250). The output was visualized as volcano plot (+BGAL1 vs. -BGAL1), annotated in PERSEUS and edited in CorelDraw.

TE vs. AF proteomics. A comparison of an in-solution-digested $N$. benthamiana apoplastic proteome (AF) versus a leaf total extract (TE) was deposited recently (6). Contaminants, reverse proteins, and those only identified by matching were removed, and majority protein groups found in three out of four biological replicates of either AF or TE were retained. $\log _{2}(\operatorname{sum} \mathrm{LFQ}$ intensity AF $+\mathrm{TE}$ ) is plotted over the distribution (sum LFQ intensity AF)/ (sum LFQ intensity AF + sum LFQ intensity TE). A ratio closer to 1 indicates predominant identification in the leaf apoplastic proteome, whereas a ratio closer to 0 indicates predominant identification in the total extract proteome.

Glycosidase activity profiling. Apoplastic fluids $(50 \mu \mathrm{l})$ were incubated in $50 \mathrm{mM}$ MES pH 5.0 with and without $1 \mu \mathrm{M} \mathrm{JJB70}$ in the dark for $1 \mathrm{~h}$ at room temperature. The labeling reaction was stopped by adding gel loading buffer (200 m M Tris- $\mathrm{HCl}$ (pH6.8), $400 \mathrm{mM}$ DTT, 8\% SDS, 0.4\% bromophenol blue, $40 \%$ glycerol) and heating at $90^{\circ} \mathrm{C}$ for $5 \mathrm{~min}$. The labeled proteins were separated on $10 \%$ gels at 200 volts for $1 \mathrm{~h}$.

Convolution ABPP. Two different samples were prepared for convolution ABPP. For the 'label-andmix' sample, apoplastic fluids isolated from PtoDC3000 $(\Delta h Q)$ infected plant $\left(1 \times 10^{6} \mathrm{CFU} / \mathrm{ml}, 2 \mathrm{dpi}\right)$ and the Mock control samples (from water infiltrated plant) were first labeled individually with JJB70 for 1 $\mathrm{h}$ at $\mathrm{pH}$ 5.0. After stopping the labeling reaction by boiling in gel loading buffer, the labeled proteomes were mixed together in equal volumes. For the 'mix-and-label' sample, equal volumes of apoplastic fluids isolated from the PtoDC3000( $\Delta h Q)$-infected and the Mock control samples were first mixed, 
preincubated for $30 \mathrm{~min}$ and then labeled with JJB70 for $1 \mathrm{~h}$ at $\mathrm{pH}$ 5.0. The labeling reaction was stopped by adding gel loading buffer and heating at $95^{\circ} \mathrm{C}$.

Detection of JJB70-labeled proteins. The fluorescently labeled proteins were detected from protein gels using the Amersham Typhoon 5 Biomolecular Imager (GE Healthcare Life Sciences, Little Chalfont, UK) using Cy2 settings (488nm excitation and 525PB20 filter). The fluorescence was quantified using ImageQuant (GE Healthcare Life Sciences, Little Chalfont, UK).

Multiplex Fluorescence ABPP. JJB70, JJB383 and FP-Tamra probes were prepared as $50 \mu \mathrm{M}, 100$ $\mu \mathrm{M}$ and $10 \mu \mathrm{M}$ stock solutions, respectively, in dimethyl sulfoxide. Labeling was performed as described previously $(10,69)$. For fluorescence gel imaging, the apoplastic fluids were incubated with $1 \mu \mathrm{M}, 2 \mu \mathrm{M}, 0.2 \mu \mathrm{M}$ probes, respectively, for $1 \mathrm{~h}$ at room temperature in the dark at a $25 \mu \mathrm{L}$ total reaction volume. The labeling reactions were stopped by adding $4 \mathrm{x}$ gel loading buffer $(200 \mathrm{~m} \mathrm{M}$ Tris$\mathrm{HCl}$ (pH6.8), $400 \mathrm{mM}$ DTT, $8 \%$ SDS, $0.4 \%$ bromophenol blue, $40 \%$ glycerol) and heating at $90^{\circ} \mathrm{C}$ for $5 \mathrm{~min}$. The labeled proteins were separated on $10 \%$ protein gels at 200 volts for $1 \mathrm{~h}$. The fluorescently JJB70, JJB383 and FP-Tamra-labeled proteins were detected from protein gels using the Amersham Typhoon-5 Biomolecular Imager (GE Healthcare Life Sciences, Little Chalfont, UK) using Cy2 settings (488nm excitation and 525PB20 filter), Cy5 settings (633nm excitation and 670PB filter) or Cy3 settings (532nm excitation and 610PB filter), respectively.

Beta-galactosidase activity assays. Apoplastic fluid samples from $N$. benthamiana leaves were incubated at $25^{\circ} \mathrm{C}$ with $0.2 \mu \mathrm{M}$ fluorescein di- $\beta$-D-galactopyranoside (FDG) (Marker Gene Technologies, Eugene, OR, USA) and $50 \mathrm{mM}$ MES buffer pH5.0 in the total reaction volume of $100 \mu \mathrm{l}$. The fluorescence of fluorescein, a product of FDG hydrolysis by $\beta$-galactosidase, was measured over time using anInfinite M200 plate reader (Tecan, Mannedorf, Switzerland) with an excitation wavelength of $485 \mathrm{~nm}$ and emission wavelength of $535 \mathrm{~nm}$.

Colorimetric assay for glycoside hydolase activities. 4-Nitrophenyl conjugates of $\beta$-Dgalactopyranoside, $\alpha$-D-galactopyranoside, $\beta$-D-mannopyranoside, $\beta$-D-glucopyranoside, $\alpha$-Dglucopyranoside, $\beta$-D-glucuronide, $\beta$-D-xylopyranoside, $\alpha$-D-xylopyranoside, $\beta$-D-fucopyranoside, $\mathrm{N}$ acetyl- $\beta$-D-glucosaminide, $\mathrm{N}$-acetyl- $\alpha$-D-glucosaminide, $\mathrm{N}$-acetyl- $\beta$-D-galactosaminide and $\mathrm{N}$-acetyl$\alpha$-D-galactosaminide (Sigma-Aldrich, Saint Louis, MO, USA) were prepared as $10 \mathrm{mM}$ stock solutions in water. Apoplastic fluids from agroinfiltrated plants were incubated with $1 \mathrm{mM}$ of each 4-Nitrophenyl conjugate of monosaccharide buffered with $50 \mathrm{mM}$ MES at pH5 for $10 \mathrm{~min}$ at $21^{\circ} \mathrm{C}$. The reactions were stopped by adding $1 \mathrm{M} \mathrm{Na}_{2} \mathrm{CO}_{3}$ and the released 4-nitrophenol was measured by monitoring absorbance at $420 \mathrm{~nm}$ using an Infinite M200 plate reader (Tecan, Mannedorf, Switzerland).

Fractionation apoplastic proteomes. Apoplastic fluids isolated from PtoDC3000 $(\Delta h Q)$ infected leaves were concentrated using vivaspin 500 spin columns with 3,000 Da MWCO filter (Sartorius Stedim Biotech, Göttingen, Germany) by centrifuging at $10,000 \mathrm{~g}, 4^{\circ} \mathrm{C}$ for $60 \mathrm{~min}$. The concentrate $(>3 \mathrm{kDa})$ and filtrate $(<3 \mathrm{kDa})$ were normalized to equal starting volumes and used for mixing and labeling assays.

Metabolite extraction. PtoDC3000 $(\Delta h Q)$ was inoculated at $\mathrm{OD}_{600}=0.5$ and grown in mannitolglutamate minimal medium supplemented with iron (70). The overnight grown bacterial culture was centrifuged and supernatant was collected. Chloroform-methanol precipitation was performed by adding $4 \mathrm{ml}$ extraction buffer (1:2.5:1 of chloroform-methanol-water) to $4 \mathrm{ml}$ supernatant or only the medium as control. The sample was vortexed and centrifuged. The upper hydrophilic (methanol/water) phase and the lower hydrophobic (chloroform) phase were collected and lyophilised. The lyophilised samples were dissolved in water and concentrated using ultrafiltration spin columns to remove the $<3 \mathrm{kDa}$ low molecular weight compounds. The filtrate fractions were collected, and treated with and without heating at $95^{\circ} \mathrm{C}$ for $5 \mathrm{~min}$.

Bacterial growth upon spray inoculation. For spray-inoculations, an overnight culture was washed and resuspended in sterile water to a density of $1 \times 10^{8} \mathrm{CFU} / \mathrm{ml}$ and sprayed onto leaf abaxial and adaxial surfaces of 3-week-old plants. Before inoculation, eight plants per genotype were incubated at $80 \%$ 
humidity for one day. Upon inoculation, plants were kept for 3-4 days in a growth cabinet at $21^{\circ} \mathrm{C}$. Three leaf discs $(1 \mathrm{~cm}$ diameter) were excised from infected leaves and each leaf disc (3.2 $\mathrm{mm}$ diameter) was soaked in $15 \% \mathrm{H}_{2} \mathrm{O}_{2}$ for 1 min to sterilise leaf surfaces. Leaf discs were washed twice in sterile water and ground in sterile water for $5 \mathrm{~min}$ using the tissuelyser and metal beads (Biospec Products, Bartelsville, OK, USA). Serial dilutions of the homogenate were plated onto KB agar plates without selection for Pta6605 strains or containing $50 \mu \mathrm{g} / \mathrm{ml}$ rifampicin for PtoDC3000 strains. Colonies were counted after $36 \mathrm{~h}$ incubation at $28^{\circ} \mathrm{C}$. The p-value was calculated using the two-tailed Student $t$-test to compare bacterial growth between WT and BGAL1 mutant plants.

Bacterial growth assay in vitro. Bacteria were harvested from an overnight preculture in LB medium, washed with sterile water and inoculated in $150 \mu \mathrm{l}$ of the specified medium with the initial $\mathrm{OD}_{600}$ of 0.05 . Bacterial growth was then monitored with the measurement of $\mathrm{OD}_{600}$ over time using Infinite M200 plate reader (Tecan, Mannedorf, Switzerland) at $28^{\circ} \mathrm{C}$ with shaking.

Isolation of flagellin proteins. Flagellin proteins were purified following the previously described procedure (28).

Oxidative burst assays. The generation of reactive oxygen species (ROS) was measured by a luminolbased assay on leaf discs adapted from (71). Luminol (Sigma-Aldrich, Saint Louis, MO, USA) was dissolved in dimethyl sulfoxide at a concentration of $10 \mathrm{mg} / \mathrm{ml}$, and kept in the dark. Horseradish peroxidase (Thermo Fisher Scientific, Waltham, MA, USA) was dissolved in water at a concentration of $10 \mathrm{mg} / \mathrm{ml}$. Leaf discs ( $6 \mathrm{~mm}$ diameter), were incubated overnight in water in a 96-well plate. Bacterial pellets of an overnight culture were washed with sterile water and resuspended to an $\mathrm{OD}_{600}$ of 0.005 and 0.05 for PtoDC3000 and Pta6605 stains in $5 \mathrm{ml}$ AF from leaves of $N$. benthamina plants transiently expressing the indicated proteins. After $1 \mathrm{~h}$ incubation at $21^{\circ} \mathrm{C}$, bacteria were centrifuged and resuspended in $2.5 \mathrm{ml}$ assay solution (water with $25 \mathrm{ng} / \mu \mathrm{l}$ luminol and $25 \mathrm{ng} / \mu \mathrm{l} \mathrm{HRP}$ ). For assays with purified flagellin, $10 \mathrm{ng} / \mu \mathrm{l}$ of flagellin were incubated in $\mathrm{AF}$ (ratio 1:15) from $N$. benthamiana leaves transiently expressing the indicated proteins for $1 \mathrm{~h} 30 \mathrm{~min}$ at RT on a rotor. After incubation, $25 \mathrm{ng} / \mu \mathrm{l}$ luminol and $25 \mathrm{ng} / \mu \mathrm{l} \mathrm{HRP}$ were added to the AF. Before measurement, the water was removed from the 96-well plate and $100 \mu \mathrm{l}$ of assay solution was added to the leaf discs. Chemiluminescence was recorded immediately using an Infinite M200 plate reader (Tecan, Mannedorf, Switzerland) every minute for 1 h. Standard errors were calculated at each time point and for each treatment. Experiments were repeated at least twice.

For protease inhibition experiments, AFs were first incubated with 1x Protease Inhibitor Cocktail (Sigma) for $1 \mathrm{~h}$ at room temperature. An overnight culture of Pta6605( $\Delta f g t l)$ was pelleted and resuspended in sterile water. Bacteria were added to the $\mathrm{AF}$ mixture to a final $\mathrm{OD}_{600}$ of 0.5 . Alternatively, flg22 peptide was added to a final concentration of $100 \mathrm{nM}$. Following a $1 \mathrm{~h}$ incubation at RT, luminol and HRP were added to the solution to final concentrations of $30 \mathrm{ng} / \mu \mathrm{l}$ and $20 \mathrm{ng} / \mu \mathrm{l}$ respectively. ROS generation was then measured as described above.

Flagellin modelling. The PstDC3000 FliC protein sequence was modelled on 5wk6 (Cryo-EM structure of $P$. aeruginosa flagellar filaments G420A, (72)) using SWISS MODEL (73) and visualised using PyMol. The best template (homo-41-mer of $P$. aeruginosa flagellin, 56.38\% sequence identity) was identified with HHblits (74) and the model was built using ProMod3 Version 1.1.0. The model quality parameters were: $\mathrm{QMQE}=0.75$ and $\mathrm{QMEAN}=-3.34$.

RT-PCR. RNA was extracted by the addition of 5 volumes of Trizol and 1 volume of chloroform to ground tissue. After brief incubation on ice, samples were separated by centrifugation and the upper aqueous phase retained. RNA was pelleted by the addition of isopropanol and centrifugation at 12,000 $g$. The pellet was washed in 70\% ethanol, air dried, and then resuspended in RNase free water. cDNA synthesis was performed using the Superscript II kit (Invitrogen, Carsbad, CA, USA). The resulting cDNA was diluted ten times and used as a template for PCR using the Mango Taq kit (Bioline, London, UK) with the primers for BGAL1 (5'atgtgacgtatgatcaccggg-3' and 5'-agcttccccaccaacttca-3') and GAPDH (5'-agctcaagggaattctcgatg-3' and 5'-aaccttaaccatgtcatctccc-3', (75)). cDNA was amplified with a $\mathrm{Tm}$ of $53^{\circ} \mathrm{C}$ for 38 cycles. 
Phylogenetic analysis. Arabidopsis and tomato GH35-domain containing proteins (PF01301) were retrieved from Genbank (Arabidopsis RefSeq proteins and NCBI Solanum lycopersicum annotation release 103). Nicotiana benthamiana GH35-domain containing proteins were extracted from the reannotated gene-models (6) and manually verified against the draft genome of $N$. benthamiana (76). Partial sequences and putative non-protein-encoding pseudogenes were cross-validated against the other available draft genomes (77). For putative pseudo-genes the closest BLAST hit in other Nicotiana species was used to visualize pseudogenization in the phylogenetic tree. Sequences were aligned with Clustal Omega (78), and the amino acid residues corresponding to BGAL1 (Nbv0.3scaffold63813_1713-9438) position 26-732 (lacking the signal peptide and the Gal-Lectin domain (PF02140)) were extracted and re-aligned using Clustal Omega. The evolutionary history was inferred by using the Maximum Likelihood method based on the Le_Gascuel_2008 model (79). The tree with the highest log likelihood (-36048.75) is shown. The percentage of trees in which the associated taxa clustered together in 1000 bootstrap repetitions is shown next to the branches. Initial tree(s) for the heuristic search were obtained automatically by applying Neighbor-Join and BioNJ algorithms to a matrix of pairwise distances estimated using a JTT model, and then selecting the topology with superior $\log$ likelihood value. A discrete Gamma distribution was used to model evolutionary rate differences among sites $(5$ categories $(+\mathrm{G}$, parameter $=1.0633)$ ). The rate variation model allowed for some sites to be evolutionarily invariable $([+\mathrm{I}], 5.50 \%$ sites $)$. The tree is drawn to scale, with branch lengths measured in the number of substitutions per site. The analysis involved 72 amino acid sequences. Evolutionary analyses were conducted in MEGA X (80).

Statistics. All values shown are mean values and the error intervals shown represent standard error of the mean (SE), unless otherwise indicated. P-values were calculated using 2-tailed Students $t$-test or Tukey HSD test, as indicated. All experiments have been reproduced and representative datasets are shown.

REFERENCES1. M. Lindeberg, Genome-enabled perspectives on the composition, evolution, and expression of virulence determinants in bacterial plant pathogens. Annu Rev Phytopathol 50, 111132 (2012).

2. T. Boller \& G. Felix. A renaissance of elicitors: perception of microbe-associated molecular patterns and danger signals by pattern-recognition receptors. Annu Rev Plant Biol 60, 379-406 (2009).

3. D. Couto \& C. Zipfel Regulation of pattern recognition signalling in plants. Nat Rev Immunol 16, 537-552 (2016)

4. H. Cui, T. Xiang, J. M. Zhou, Plant immunity: a lesson from pathogenic bacteria effector proteins. Cell Microbiol 11, 1453-1461 (2009).

5. J. Fliegmann, G. Felix, Immunity: flagellin seen from all sides. Nat Plants 2, 16136 (2016).

6. J. Kourelis et al., Re-annotated Nicotiana benthamiana gene models for enhanced proteomics and reverse genetics. BioRxiv 2018 (https://doi.org/10.1101/373506).

7. F. Grosse-Holz et al., The transcriptome, extracellular proteome and active secretome of agroinfiltrated Nicotiana benthamiana uncover a large, diverse protease repertoire. Plant Biotechnol J 16, 1068-1084 (2018).

8. C. F. Wei et al., A Pseudomonas syringae pv. tomato DC3000 mutant lacking the type III effector HopQ1-1 is able to cause disease in the model plant Nicotiana benthamiana. Plant J 51, 32-46 (2007). 
9. W. W. Kallemeijn et al., Novel activity-based probes for broad-spectrum profiling of retaining $\beta$ exoglucosidases in situ and in vivo. Angew Chem Int Ed 51, 12529-12533 (2012).

10. B. Chandrasekar et al., Broad-range glycosidase activity profiling. Mol Cell Proteomics 13, 27872800 (2014).

11. B. Chandrasekar, T. N. Hong, R. A. L. van der Hoorn, Inhibitor discovery by convolution ABPP. Methods Mol Biol 1491, 47-56 (2017).

12. V. Nekrasov, B. Staskawicz, D. Weigel, J. D. Jones, S. Kamoun, Targeted mutagenesis in the model plant Nicotiana benthamiana using Cas9 RNA-guided endonuclease. Nat Biotechnol 31, 691-693 (2013).

13. S. Ranf et al., A lectin S-domain receptor kinase mediates lipopolysaccharide sensing in Arabidopsis thaliana. Nat Immunol 16, 426-433 (2015).

14. A. Miya et al., CERK1, a LysM receptor kinase, is essential for chitin elicitor signaling in Arabidopsis. Proc Natl Acad Sci USA 104, 19613-19618 (2007).

15. R. Willmann et al., Arabidopsis lysin-motif proteins LYM1 LYM3 CERK1 mediate bacterial peptidoglycan sensing and immunity to bacterial infection. Proc Natl Acad Sci USA 108, 1982419829 (2011).

16. D. Couto, C. Zipfel, Regulation of pattern recognition receptor signalling in plants. Nat Rev Immunol 16, 537-552 (2016).

17. L. Gómez-Gómez, T. Boller, FLS2: an LRR receptor-like kinase involved in the perception of the bacterial elicitor flagellin in Arabidopsis. Mol Cell 5, 1003-1011 (2000).

18. C. Segonzac et al., Hierarchy and roles of pathogen-associated molecular pattern-induced responses in Nicotiana benthamiana. Plant Physiol 156, 687-699 (2011).

19. B. H. Kvitko et al., Deletions in the repertoire of Pseudomonas syringae pv. tomato DC3000 type III secretion effector genes reveal functional overlap among effectors. PLoS Pathog 5, e1000388 (2009).

20. G. Felix, J. D. Duran, S. Volko, T. Boller, Plants have a sensitive perception system for the most conserved domain of bacterial flagellin. Plant J 18, 265-276 (1999).

21. S. Robatzek et al., Molecular identification and characterization of the tomato flagellin receptor LeFLS2, an orthologue of Arabidopsis FLS2 exhibiting characteristically different perception specificities. Plant Mol Biol 64, 539-547 (2007).

22. D. R. Hann, J. P. Rathjen, Early events in the pathogenicity of Pseudomonas syringae on Nicotiana benthamiana. Plant J 49, 607-618 (2007).

23. R. Takai, A. Isogai, S. Takayama, F. S. Che, Analysis of flagellin perception mediated by flg22 receptor OsFLS2 in rice. Mol Plant Microbe Interact 21, 1635-1642 (2008).

24. Y. Rossez, E. B. Wolfson, A. Holmes, D. L. Gally, N. J. Holden, Bacterial flagella: twist and stick, or dodge across the kingdoms. PLoS Pathog 11, e1004483 (2015).

25. K. Chiku et al., Comparative analysis of flagellin glycans among pathovars of phytopathogenic Pseudomonas syringae. Carbohydr Res 375, 100-104 (2013).

26. M. Yamamoto et al., Identification of genes involved in the glycosylation of modified viosamine of flagellins in Pseudomonas syringae by Mass Spectrometry. Genes 2, 788-803 (2011). 
27. S. Lee, D. S. Yang, S. R. Uppalapati, L. W. Sumner, K. S. Mysore, Suppression of plant defense responses by extracellular metabolites from Pseudomonas syringae pv. tabaci in Nicotiana benthamiana. BMC Plant Biol 13, 65 (2013).

28. F. Taguchi et al., Identification of glycosylation genes and glycosylated amino acids of flagellin in Pseudomonas syringae pv. tabaci. Cell Microbiol 8, 923-938 (2006).

29. L. C. Nguyen et al., Genetic analysis of genes involved in synthesis of modified 4-amino-4,6dideoxyglucose in flagellin of Pseudomonas syringae pv. tabaci. Mol Genet Genomics 282, 595605 (2009).

30. B. A. Vinatzer et al., The type III effector repertoire of Pseudomonas syringae pv. syringae B728a and its role in survival and disease on host and non-host plants. Mol Microbiol 62, 26-44 (2006).

31. S. R. Hind et al., Tomato receptor flagellin-sensing 3 binds flgII-28 and activates the plant immune system. Nat Plants 2, 1-8 (2016).

32. Y. Katsuraghi et al., CD2-1, the C-terminal region of flagellin, modulates the induction of immune repsonses in rice. Mol Plant-Microbe Interact 28, 648-58 (2015).

33. F. Brunner et al., Pep-13, a plant defense-inducing pathogen-associated pattern from Phytophthora transglutaminases. EMBO J 21, 6681-6688 (2002).

34. S .Oome et al., Nep1-like proteins from three kingdoms of life act as a microbe-associated molecular pattern in Arabidopsis. Proc Natl Acad. Sci USA 111, 16955-16960 (2014).

35. C. Ottmann et al., A common toxin fold mediates microbial attack and plant defense. Proc Natl Acad Sci USA 106, 10359-10364 (2009).

36. K. Reiss et al., Structural and phylogenetic analyses of the GP42 transglutaminase from Phytophthora sojae reveal an evolutionary relationship between oomycetes and marine Vibrio bacteria. J Biol Chem 286, 42585-42593 (2001).

37. H. Böhm et al. A conserved peptide pattern from a widespread microbial virulence factor triggers pattern-induced immunity in Arabidopsis. PLoS Pathog 10, e1004491 (2004).

38. H. Hirai et al., Glycan moiety of flagellin in Acidovorax avenae $\mathrm{K} 1$ prevents the recognition by rice that causes the induction of immune responses. Plant Signal Behav 9, e972782 (2014).

39. F. Taguchi et al., Post-translational modification of flagellin determines the specificity of HR induction. Plant Cell Physiol 44, 342-349 (2003).

40. K. Takeuchi et al., Flagellin glycans from two pathovars of Pseudomonas syringae contain rhamnose in $\mathrm{D}$ and $\mathrm{L}$ configurations in different ratios and modified 4-amino-4,6-dideoxyglucose. J Bacteriol 189, 6945-6956 (2007).

41. S. K. Arora, A. N. Neely, B. Blair, S. Lory, R. Ramphal, Role of motility and flagellin glycosylation in the pathogenesis of Pseudomonas aeruginosa burn wound infections. Infect Immun 73, 4395-4398 (2005).

42. A. Hanuszkiewicz et al., Identification of the flagellin glycosylation system in Burkholderia cenocepacia and the contribution of glycosylated flagellin to evasion of human innate immune responses. J Biol Chem 289, 19231-19244 (2014).

43. C. Schäffer, P. Messner, Emerging facets of prokaryotic glycosylation. FEMS Microbiol Rev 41, 49-91 (2017). 
44. A. E. Scott et al., Flagellar glycosylation in Burkholderia pseudomallei and Burkholderia thailandensis. J Bacteriol 193, 3577-3587 (2011).

45. T. N. Petersen, S. Brunak, G. von Heijne, H. Nielsen, SignalP 4.0: discriminating signal peptides from transmembrane regions. Nat Methods 8, 785-786 (2011).

46. S. Gimenez-Ibanez et al., AvrPtoB targets the LysM receptor kinase CERK1 to promote bacterial virulence on plants. Curr Biol 19, 423-429 (2009).

47. A. Heese et al., The receptor-like kinase SERK3/BAK1 is a central regulator of innate immunity in plants. Proc Natl Acad Sci USA 104, 12217-12222 (2007).

48. M. Gao et al., Regulation of cell death and innate immunity by two receptor-like kinases in Arabidopsis. Cell Host Microbe 6, 34-44 (2009).

49. C. Zipfel et al., Bacterial disease resistance in Arabidopsis through flagellin perception. Nature 428, 764-767 (2004).

50. C. Waite, J. Schumacher, M. Jovanovic, M. Bennett, M. Buck, Negative autogenous control of the master type III secretion system regulator hrpL in Pseudomonas syringae. MBio 8, (2017).

51. W. Wei et al., The gene coding for the Hrp pilus structural protein is required for type III secretion of Hrp and Avr proteins in Pseudomonas syringae pv. tomato. Proc Natl Acad Sci USA 97, 22472252 (2000).

52. R. Shimizu et al., The DeltafliD mutant of Pseudomonas syringae pv. tabaci, which secretes flagellin monomers, induces a strong hypersensitive reaction (HR) in non-host tomato cells. Mol Genet Genomics 269, 21-30 (2003).

53. H. Feil et al., Comparison of the complete genome sequences of Pseudomonas syringae pv. syringae B728a and pv. tomato DC3000. Proc Natl Acad Sci USA 102, 11064-11069 (2005).

54. R. A. L. Van der Hoorn, S. Rivas, B. B. Wulff, J. D. Jones, M. H. Joosten, Rapid migration in gel filtration of the Cf-4 and Cf-9 resistance proteins is an intrinsic property of $\mathrm{Cf}$ proteins and not because of their association with high-molecular-weight proteins. Plant J 35, 305-315 (2003).

55. Y. Liu, M. Schiff, R. Marathe, S. P. Dinesh-Kumar, Tobacco Rar1, EDS1 and NPR1/NIM1 like genes are required for $N$-mediated resistance to tobacco mosaic virus. Plant J 30, 415-429 (2002).

56. M. Stemmer, T. Thumberger, M. Del Sol Keyer, J. Wittbrodt, J. L. Mateo, CCTop: An intuitive, flexible and reliable CRISPR/Cas9 target prediction tool. PLoS One 10, e124633 (2015).

57. J. G. Doench et al., Optimized sgRNA design to maximize activity and minimize off-target effects of CRISPR-Cas9. Nat Biotechnol 34, 184-191 (2016).

58. E. Weber, C. Engler, R. Gruetzner, S. Werner, S. Marillonnet, A modular cloning system for standardized assembly of multigene constructs. PLoS One 6, e16765 (2011).

59. K. Belhaj, A. Chaparro-Garcia, S. Kamoun, V. Nekrasov, Plant genome editing made easy: targeted mutagenesis in model and crop plants using the CRISPR/Cas system. Plant Methods 9, 39 (2013).

60. I. A. Sparkes, J. Runions, A. Kearns, C. Hawes, Rapid, transient expression of fluorescent fusion proteins in tobacco plants and generation of stably transformed plants. Nat Protoc 1, 2019-2025 (2006).

61. F. Grosse-Holz et al., Three unrelated protease inhibitors enhance accumulation of pharmaceutical recombinant proteins in Nicotiana benthamiana. Plant Biotechnol J 16, 1797-1810 (2018). 
62. J. Rappsilber, M. Mann, Y. Ishihama, Protocol for micro-purification, enrichment, prefractionation and storage of peptides for proteomics using StageTips. Nat Protoc 2, 1896-1906 (2007).

63. A. Michalski et al., Ultra high resolution linear ion trap Orbitrap mass spectrometer (Orbitrap Elite) facilitates top down LC MS/MS and versatile peptide fragmentation modes. Mol Cell Proteomics 11, O111.013698 (2012).

64. J. V. Olsen et al., Parts per million mass accuracy on an Orbitrap mass spectrometer via lock mass injection into a C-trap. Mol Cell Proteomics 4, 2010-2021 (2005).

65. J. Cox et al., Andromeda: a peptide search engine integrated into the MaxQuant environment. $J$ Proteome Res 10, 1794-1805 (2011).

66. J. Cox, M. Mann, MaxQuant enables high peptide identification rates, individualized p.p.b.-range mass accuracies and proteome-wide protein quantification. Nat Biotechnol 26, 1367-1372 (2008).

67. J. Cox et al., Accurate proteome-wide label-free quantification by delayed normalization and maximal peptide ratio extraction, termed MaxLFQ. Mol Cell Proteomics 13, 2513-2526 (2014).

68. S. Tyanova et al., The Perseus computational platform for comprehensive analysis of (prote)omics data. Nat Methods 13, 731-740 (2016).

69. A. M. Husaini et al., Multiplex fluorescent activity profiling of $\alpha$-glycosidases in plants. Plant Physiol 177, 24-37 (2018).

70. P. A. Bronstein et al., Global transcriptional responses of Pseudomonas syringae DC3000 to changes in iron bioavailability in vitro. BMC Microbiol 8, 209 (2008).

71. J. M. Smith, A. Heese, Rapid bioassay to measure early reactive oxygen species production in Arabidopsis leave tissue in response to living Pseudomonas syringae. Plant Methods 10, 6 (2014).

72. F. Wang et al., A structural model of flagellar filament switching across multiple bacterial species. Nat Commun 8, 960 (2017).

73. A. Waterhouse et al., SWISS-MODEL: homology modelling of protein structures and complexes. Nucleic Acids Res 46, W296-W303 (2018).

74. M. Remmert, A. Biegert, A. Hauser, J. Söding, HHblits: lightning-fast iterative protein sequence searching by HMM-HMM alignment. Nat Methods 9, 173-175 (2011).

75. D. Liu et al., Validation of reference genes for gene expression studies in virus-infected Nicotiana benthamiana using quantitative real-time PCR. PLoS One 7, e46451 (2012).

76. A. Bombarely et al., A draft genome sequence of Nicotiana benthamiana to enhance molecular plant-microbe biology research. Mol Plant-Microbe Interact 25, 1523-1530 (2012).

77. F. Naim et al., Advanced engineering of lipid metabolism in Nicotiana benthamiana using a draft genome and the V2 viral silencing-suppressor protein. PLoS One 7, e52717 (2012).

78. F. Sievers et al., Fast, scalable generation of high-quality protein multiple sequence alignments using Clustal Omega. Mol Syst Biol 7, 539 (2011).

79. S. Q. Le, O. Gascuel, An improved general amino acid replacement matrix. Mol Biol Evol 25, 1307-1320 (2008).

80. S. Kumar, G. Stecher, M. Li, C. Knyaz, K. Tamura, MEGA X: Molecular evolutionary genetics analysis across computing platforms. Mol Biol Evol 35, 1547-1549 (2018). 